\title{
Individualized closed-loop anesthesia through patient model partitioning
}

\author{
Ylva Wahlquist
}

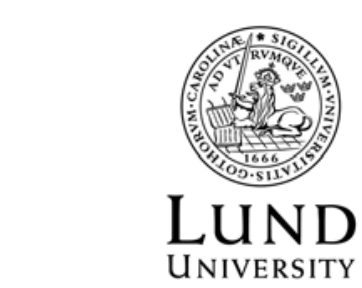

Department of Automatic Control 
MSc Thesis

TFRT-6080

ISSN 0280-5316

Department of Automatic Control

Lund University

Box 118

SE-221 00 LUND

Sweden

(C) 2019 by Ylva Wahlquist. All rights reserved.

Printed in Sweden by Tryckeriet i E-huset

Lund 2019 


\section{Abstract}

This master thesis project proposes methods for individualizing closed-loop controlled anesthesia. One of the largest challenges with closed-loop anesthesia is the variation between patients in the sensitivity to the anesthetic drug, here propofol. Due to limited excitation in the process dynamics together with a high measurement noise level is it not possible to determine a full reliable model describing a patient's dynamics online. The method used here for minimizing the effects of inter-patient variability was through patient model partitioning of children and adult models. Partitioning was based on similarity measures between patients, for example age, weight and applied to a dynamic model describing each patient. For each subset resulting from partitioning, an optimal PID controller has been synthesized. This thesis has shown that the effects of inter-patient variability can be reduced using partitioning into two subsets. More subsets did not result in a significant reduction. Partitioning based on $v$-gap between patient models resulted in the best attenuation of surgical stimulation disturbances. Partitioning based on age for children and weight for adults reduces the impact from surgical stimulation were proposed for clinical practices. These methods are easy to implement because the demographics are known beforehand and does not depend on actual measurements during the anesthesia. The results are substantiated by simulations and calculations of achieved attenuation with acceptable performance and preserved robustness. 



\section{Acknowledgements}

I would first like to thank my supervisor Kristian Soltesz. I am very grateful to you for introducing me to this interesting research field. You have always come with valuable inputs on my proceeding work and I am very grateful for all of your help.

In the research group at BCCHR, University of British Columbia, Vancouver, Canada, I must acknowledge professor Guy Dumont and Dr. Klaske van Heusden for letting me visit and take part of their work at the Childrens Hospital of British Columbia. I would also like to thank Dr. Mark Ansermino for letting me visit anesthesia surgeries during my stay, giving me a broader perspective of the subject. The rest of the research group requires an acknowledgement for the warm welcome during my stay in Vancouver.

I also want to thank researcher Richard Pates for the introduction and help with implementation of the $v$-gap.

A special thank to $\mathrm{PhD}$ student José Manuel González Cava who has provided me with the code to the synthesis, particularly designed for the children model set. He has also been very helpful during the project, always available for questions. 



\section{Contents}

1. Introduction 9

1.1 Closed-loop controlled anesthesia . . . . . . . . . . . . . . 9

1.2 Problem formulation . . . . . . . . . . . . . . . . . . . . 10

1.3 Anesthesia . . . . . . . . . . . . . . . . 11

2. Patient models 13

2.1 PKPD model structure . . . . . . . . . . . . . . . . . 13

2.2 Patient models from clinical data . . . . . . . . . . . . . . 14

2.3 Delay . . . . . . . . . . . . . . . . . . . . 15

2.4 Inter-patient variability $\ldots \ldots \ldots \ldots \ldots$

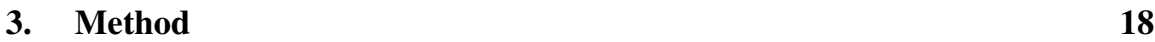

3.1 Partitioning . . . . . . . . . . . . . . . 18

3.2 Partitioning based on physical characteristics . . . . . . . . . . 19

3.3 Partitioning based on delay . . . . . . . . . . . . . . . . . . 19

3.4 Partitioning based on on $v$-gap $\ldots \ldots \ldots \ldots \ldots \ldots$

3.5 Controller structure . . . . . . . . . . . . . . . . 26

3.6 Controller synthesis . . . . . . . . . . . . . . . . . . . 28

4. Results 30

4.1 Initial controller and best case . . . . . . . . . . . . . 30

4.2 Partitioning based on physical characteristics . . . . . . . . . 36

4.3 Partitioning based on delay . . . . . . . . . . . . 38

4.4 Partitioning based on $v$-gap . . . . . . . . . . . 43

5. Discussion 48

5.1 Limitations . . . . . . . . . . . . . . . . . . 50

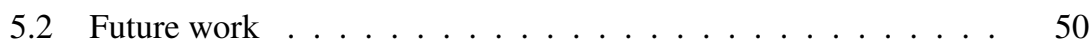

6. Conclusion $\quad 52$

Bibliography $\quad 53$

Nomenclature $\quad 56$ 



\section{1}

\section{Introduction}

\subsection{Closed-loop controlled anesthesia}

Anesthesia is a state of controlled unconsciousness. It is used during surgeries and in intensive care units when the procedure would be too painful or otherwise inappropriate for the patient to be awake. Anesthesia reduces the sensing abilities so that the patient cannot feel pain or touch and will not remember the surgery afterwards. During surgery, it is important to keep the patient unconscious through the entire surgery. The anesthesia itself is not without risk due to possible over- and underdosing of anesthetic drugs, even if severe complications are rare.

Traditionally, the drug dosing is manually controlled by the anesthesiologist. The anesthesiologist estimates the anesthetic state and adjusts the drug dosage accordingly. The anesthetic state is estimated by looking at the patients movements, breathing and hemodynamics, for example heart rate. A correct drug dosage is of importance because under-dosing may lead to awareness and over-dosing may lead to longer emergence phase, possible side effects such as nausea or adverse hemodynamic response. Deep anesthesia may also lead to apnea if the procedure requires access to the airways so that the patient can not be put on respirator.

In this thesis, closed-loop control of anesthesia is considered. Closed-loop control means that the drug dosing is based on feedback from a measurement-based estimate of the anesthetic state. A schematic view of closed-loop controlled anesthesia is shown in Figure 1.1. The electroencephalogram (EEG) of the patient is continuously measured and depending on the EEG, a digital controller calculates an appropriate drug infusion rate for the infusion pump to give the patient.

This methodology was introduced in the 1950s by a group lead by Bickford. One study where the EEG was analyzed for abdominal surgery is [Mayo et al., 1950]. Since then, there has been research involving clinical evaluation of closedloop controlled anesthesia [van Heusden et al., 2014], [Liu et al., 2011] and [Struys et al., 2001].

One of the largest challenges with closed-loop anesthesia is the inter-patient variability, meaning that patients react differently to infusions of anesthetic drugs. This depends for example on variation in the metabolism between patients [van 


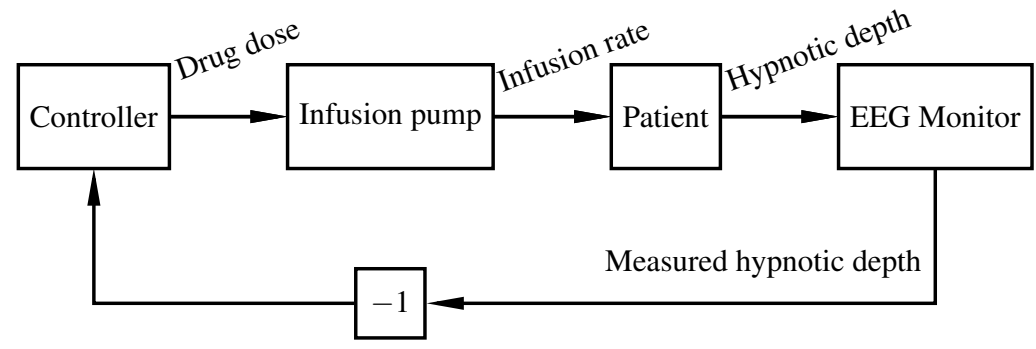

Figure 1.1: A schematic overview of closed-loop anesthesia where the depth of consciousness is measured with EEG and the drug dose determined by the controller.

Heusden et al., 2013]. This problem was looked into in [Soltesz et al., 2016], from where this thesis has its starting point.

\subsection{Problem formulation}

The main problem and purpose of this thesis was to minimize the effects of the inter-patient variability of patients for safe and adequate anesthesia. Closed-loop controlled anesthesia can reduce the effects of the inter-patient variability by reducing possible under- and over-dosing. If the inter-patient variability is not taken into account, closed-loop control may show undesirable oscillatory behaviour or result in an overshoot during induction of anesthesia. When tuning the controller parameters, one must also take into account that induction needs to be fast enough to not slow down the surgical procedure. This thesis proposes a method to minimize the effects of inter-patient variability through patient model partitioning. It is wanted that the partition functions so that patients with similar dynamics belong to the same subset. A controller was then synthesized for each subset.

A patient's response to a drug infusion is unknown before the anesthesia. Due to this, a conservative controller is normally chosen to make sure that even a sensitive patient was safely anesthetized. During anesthesia it is possible to estimate the actual dynamics of a patient, using the drug infusion profile and associated EEGbased hypnotic depth measure. It is preferable to identify the dynamics during the first phase of anesthesia (called induction, described further in Section 1.3) when the (surgical) disturbance level is relatively low and where there is a control signal that excites the process dynamics. Due to limited excitation it is generally not possible to obtain a full model describing the patients dynamics from the induction phase data. If it would be possible, an individual controller could be chosen to maximize performance and robustness for this patient.

In this thesis, we make use of a set of patient models that were previously identified from clinical data in [van Heusden et al., 2013] and [van Heusden et al., 2017]. These models are described in detail in Chapter 2. A robust, but conservative, con- 
troller has earlier been designed for the patients in [van Heusden et al., 2014] for the children model set and in [Dumont et al., 2011] for the adult model set.

Through partitioning of the the patient model set into subsets with mutually similar dynamics, a controller was synthesized for each subset. Controllers was synthesized with constraints so that the resulting controllers have at least the same performance and maintained robustness compared to a nominal controller originally designed for the children model set. Several questions need to be addressed:

- Which metric should be used to describe similarity between patients?

- How should partitioning be performed?

- If we perform partitioning depending on induction phase data, how can we design a robust classifier which can be used online after the induction phase for a given patient?

\subsection{Anesthesia}

Anesthesia is a state of unconsciousness, coming from the Greek word $\alpha \nu \alpha \imath \sigma \theta \eta \sigma \imath \alpha$, "anaisthēsis", meaning "without sensation". It involves not only a state of controlled unconsciousness, but also lack of pain reception, loss of recall (amnesia) and muscle relaxation [Bibian et al., 2005].

Thus, anesthesia can be divided into three components: hypnosis, analgesia and neuromuscular blockade. The main part is hypnosis, meaning temporary druginduced loss of consciousness and memory. A temporary loss of memory is particularly important if a patient is awakening and feels pain during surgery [Ranta et al., 1998]. This is rare and highly undesirable. Hypnosis is commonly achieved with intravenous infusions of the drug propofol. This thesis considers control of the hypnotic state using propofol.

The second component is analgesia. Analgesic drugs affect the nervous system, giving the patient sensation loss, relieving pain in the beginning, during and after the surgery. An analgesic drug is often co-administered together with a hypnotic drug. In the clinical trials [van Heusden et al., 2013] and [van Heusden et al., 2017] from where this thesis has received its data, the opioid named remifentanil is used together with propofol. The third component is neuromuscular blockade which is a state of muscle relaxation and is not considered in this thesis.

A typical anesthetic episode can be divided into three phases. The first phase is the induction phase where the patient is brought to unconsciousness. Normally, particularly for children, a bolus (large drug dose over short time) is given to induce anesthesia. The induction phase is normally a few minutes long. It is desirable with a short induction time to minimize patient discomfort and to be resource efficient. The drugs increase the depth of hypnosis (DOH) [Zikov et al., 2006] to a desired and stable level, which is controlled throughout the maintenance phase. Surgery takes 
place during this phase. Surgical stimuli reduce the depth of hypnosis so that the patient becomes more conscious and can be viewed as output disturbances [Bibian et al., 2005]. The final phase is the emergence phase where the drug infusions are terminated and the patient wakes up. A long emergence phase may lead to nausea and other side effects. The DOH varies during the surgery, in particularly when there is a surgical disturbance. The disturbance is immeasurable and must be counteracted by the anesthesiologist.

The level of consciousness is denoted DOH, depth of hypnosis. It can be estimated using the electroencephalogram, EEG. The EEG is analyzed to yield a level of consciousness measure. One commonly used DOH index is the bispectral index, BIS. BIS is based on bispectral analysis which divides the EEG data into epochs and computes the Fourier transform of each epoch, as further explained in [Sigl and Chamoun, 1994]. The BIS is reported on a scale between 100 and 0 where $90-100$ represents full wakefulness and 0 represents an iso-electric EEG. Another index that makes use of the same scale is $\mathrm{WAV}_{\mathrm{CNS}}$, derived from wavelet coefficients in the EEG signals [Sadati et al., 2018]. The $\mathrm{WAV}_{\mathrm{CNS}}$ index is provided by the NeuroSense NS-701 monitor (NeuroWave Systems Inc., Cleveland Heights, OH) [Zikov et al., 2006]. This index is used in this thesis and will be referred to as depth of hypnosis or DOH. A value between 40 and 60 is considered appropriate for many surgical procedures. 


\section{2}

\section{Patient models}

To be able to develop and evaluate better controllers for automation in anesthesia, models derived from clinical data are used. These models describe how a patient responds to a drug and was be used for controller design. One important thing to keep in consideration is that patients that were difficult to model are not part of the patient model set and therefore not part of this study.

\subsection{PKPD model structure}

A patient can be modeled with a pharmacokinetic (PK) and a pharmacodynamic (PD) model. The model structure can be seen in Figure 2.1. Together, they describe how the drug infusion rate $u(t)$ relates to the clinical effect $E(t)$, which is measured by the EEG monitor. The PK model describes how the body takes up, distributes and eliminates the drug, or how the drug infusion rate relates to the plasma concentration $C_{p}(t)$. The PD model describes how the plasma concentration is related to the clinical effect. When combined, these are referred to as a PKPD model.

The PK part of the model is derived from a population average model in literature. The PD model consists of two parts: a linear first-order transfer function with a time delay together with a nonlinearity. The first part relates the plasma concentration, $C_{p}(t)$, and the concentration at the effect site (i.e., the brain), $C_{e}(t)$, and can

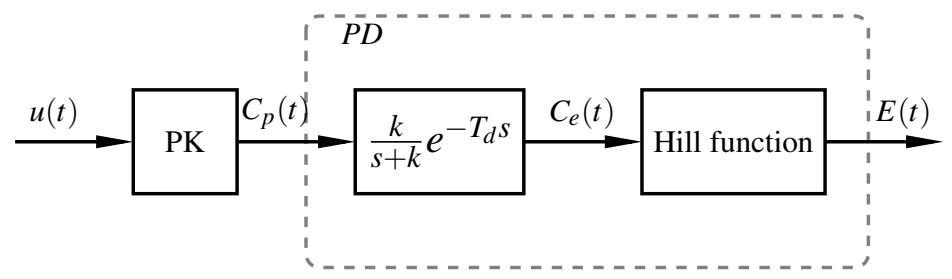

Figure 2.1: A PKPD model structure describing relationship between drug infusion rate and clinical effect. The PD model consists of two parts, a linear first-order transfer function with delay and a nonlinear Hill function. 
be described as,

$$
G_{C_{e}, C_{p}}(s)=\frac{k}{s+k} e^{-T_{d} s},
$$

where the gain $k$ and delay $T_{d}$ are identified from data. The nonlinearity is the Hill function, parameterized as in [van Heusden et al., 2013], relating $C_{e}(t)$ and the clinical effect $E(t)$ (measured in $\mathrm{WAV}_{\mathrm{CNS}}$ units)

$$
E(t)=E_{0}-E_{0} \frac{C_{e}^{\gamma}(t)}{E C_{50}^{\gamma}+C_{e}^{\gamma}(t)},
$$

where $E C_{50}$ is $50 \%$ clinical of the maximal clinical effect and $\gamma$ is the "Hill parameter". $E_{0}$ is the clinical effect in absence of a drug, scaled as $100 \geq E_{0}>0$. A detailed explanation of how the parameters can be identified from data is given in [Bibian et al., 2006] and [van Heusden et al., 2013].

\subsection{Patient models from clinical data}

Models for both children and adults are used in this thesis. They were initially published in [van Heusden et al., 2013] and [van Heusden et al., 2017], respectively. The clinical data from where the models are derived comes from both manually administered and closed-loop controlled anesthesia. Originally the models are nonlinear (see (2.2)) but they are linearized around the operating point corresponding to $50 \mathrm{WAV}_{\mathrm{CNS}}$ and discretized for design.

The models are represented as discrete time LTI state space systems. There are 47 children models from [van Heusden et al., 2013] and 31 adult models from [van Heusden et al., 2017]. In addition to the PKPD models, information about age, gender, body weight and body height is available for each modeled patient, see Table 2.1. Other information available with the models is the clinical effect in absence of a drug, $E_{0}$. Estimation of $E_{0}$ has earlier been made from the first 50 seconds of induction data. The adult models also contain information about LBM, lean body mass.

For adults the use of body mass may lead to overdosing for obese patients, therefore another weight indicator is used, LBM. LBM is a measure of difference between total body weight and body fat. Normally, it averages between $60 \%$ and $90 \%$ of total body weight [Hume, 1966]. An estimation of a LBM can be computed from height and weight. The following definition from [Hume, 1966] of LBM is used

$$
\begin{aligned}
& \text { Male: } \\
& \mathrm{LBM}=0.32810 \mathrm{bwt}+0.33929 \mathrm{bht}-29.5336 \\
& \text { Female: } \\
& \mathrm{LBM}=0.29569 \mathrm{bwt}+0.41813 \mathrm{bht}-43.2933 \text {, }
\end{aligned}
$$

where bwt is weight in $\mathrm{kg}$, and bht the height in $\mathrm{cm}$. 
Table 2.1: Demographic data for the models sets, value(range). LBM denotes lean body mass with definition from [Hume, 1966].

\begin{tabular}{ccc}
\hline & Children, $\mathrm{n}=47$ & Adults, $\mathrm{n}=31$ \\
\hline Age $(\mathrm{yr})$ & $12(6-16)$ & $61(38-82)$ \\
Gender $(\mathrm{F} / \mathrm{M})$ & $26 \mathrm{~F}, 19 \mathrm{M}$ & $13 \mathrm{~F}, 18 \mathrm{M}$ \\
Height $(\mathrm{cm})$ & $155.4(119-181)$ & $173(155-190)$ \\
Weight $(\mathrm{kg})$ & $47.3(21-82)$ & $84(47-118)$ \\
$E_{0}$ & $91.4(87-95)$ & $91.6(87.8-94.0)$ \\
LBM $(\mathrm{kg}$, only adults $)$ & - & $55.8(38.3-68.4)$ \\
\hline
\end{tabular}

\subsection{Delay}

It is known that PKPD models display a large variation in phase lag between patients [van Heusden et al., 2013]. This lag can be represented as a delay, denoted $T_{d}$ (see (2.1), in low-order models. A long model time delay $T_{d}$ limits the performance of the controller and makes it more conservative.

In the estimation of delay, there was a trade-off between the delay, the parameter $k$ and the non-linearity $\gamma$, in (2.1) and (2.2). This comes from difficulties in uniquely identifying gamma and $T_{d}$ simultaneously, provided the excitation in representative induction phase data [van Heusden et al., 2013]. To guarantee robustness, a choice was made to overestimate the delay and consequently underestimate gamma. For some children, the $\mathrm{WAV}_{\mathrm{CNS}}$ index increased in the beginning of the induction phase which may depend on pain during injection. This could also lead to an overestimation of the delay. Due to possibility in overestimation, the delay is limited to $T_{d}<120 \mathrm{~s}$ for children. During anesthesia, the delay is most visible during the induction phase, when the input essentially undergoes a step change.

The delay varies a lot between patient models and does not depend on age, gender etc, see Figure 2.2 for dependence between delay and physical demographics in the children data set. The adult data set yields similar appearance. 
Chapter 2. Patient models
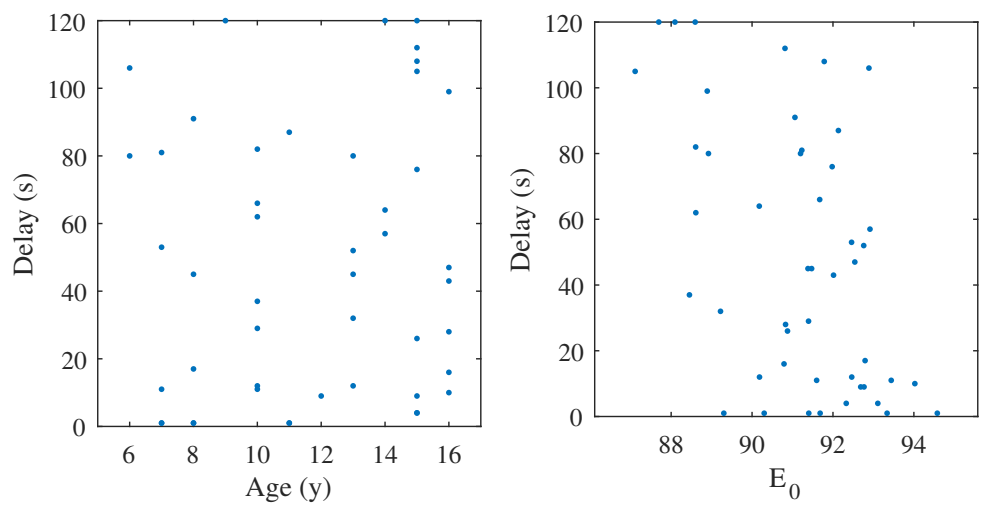

(a) Age, $R^{2}=0.0090$

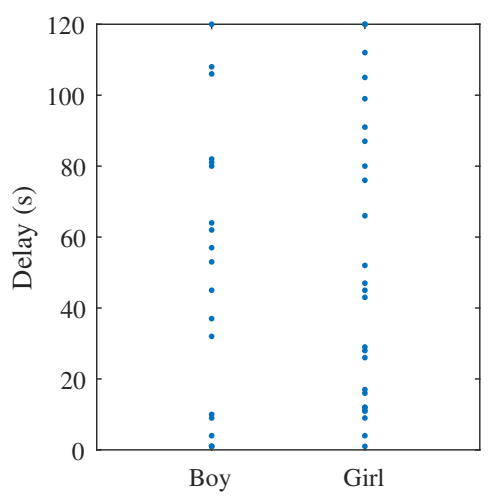

(b) $E_{0}, R^{2}=0.2512$

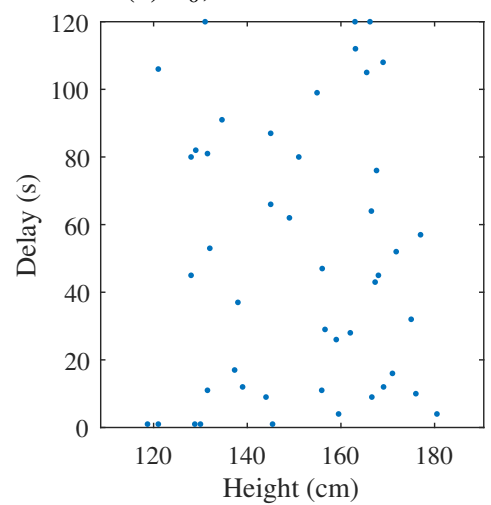

(c) Gender

(d) Height, $R^{2}=8.91 \cdot 10^{-4}$

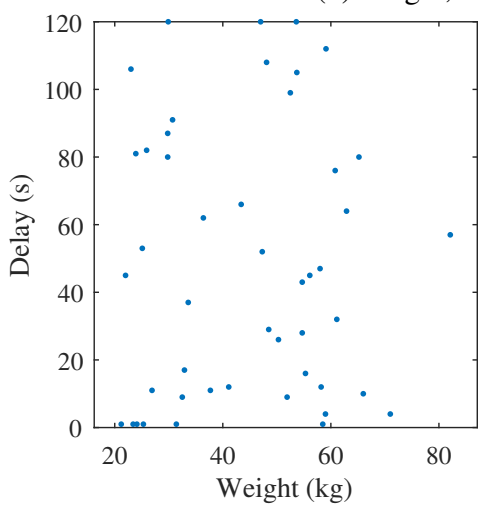

(e) Weight, $R^{2}=4.80 \cdot 10^{-4}$

Figure 2.2: Dependence between delay $T_{d}$ and physical demographics. The model set is children 6-16 y. A linear regression has been made for the data in each plot, where the $R^{2}$ value is shown in the subcaption. 


\subsection{Inter-patient variability}

The response to propofol displays a large variation between patients. The variability comes from differences in distribution and elimination of the drug in the body, also influenced by the patient's age, body mass, liver function etc. [Bibian et al., 2005].

To guarantee robustness, a conservative feedback controller is required. Else, the inter-patient variability may lead to over- or underdosing of anesthetic drugs for or result in undesirable oscillatory behaviour [van Heusden et al., 2014]. Closed-loop anesthesia must be able to safely handle the inter-patient variability. The variation comes from differences in the pharmacokinetics and pharmacodynamics between patients. Difference in PD shows that some patients require a larger plasma concentration before reaching a certain clinical effect.

As mentioned in the previous section, full models are generally not automatically identifiable from induction phase data. If they were, the inter-patient variability would not be a problem and could be taken into account. 


\section{3}

\section{Method}

We propose a method of minimizing the effects of inter-patient variability through patient model partitioning. In this thesis, this will be handled in the following way:

1. Partitioning of the patient model set into subsets through clustering.

2. Design one controller for each subset, which maximizes performance while ensuring robustness over the subset.

3. When a new patient is to be anesthetized, determine which subset the patient belongs to by either given a priori demographic information or through the induction phase response using a conservative controller and subsequently switch to the controller associated with that subset.

Clinical induction data is used for analysis together with the patient models. The clinical data consists of actual infusion rates and the corresponding measured depth of hypnosis during surgery from studies resulting in [van Heusden et al., 2013] and [van Heusden et al., 2017]. Information about protocols and ethics appeal can be found in these articles. This clinical induction data is not presented directly in the thesis but used for evaluation.

\subsection{Partitioning}

The main objective of this thesis is to make a suitable partition of an initial patient model set. For example, if patient $A$ should be anesthetized and if patient $A$ responds similarly to patient $B$ who has been anesthetized earlier on, then patient $A$ should have similar controller settings to $B$. The methods described here were developed and evaluated using two model sets described in Chapter 2 comprising 47 models of children and 31 models of adults. The patient models are clustered into two or more subsets, and those in the same subset have the same controller settings. The goal is that these controllers should perform better for patient models in the corresponding subset than the corresponding controller synthesized for the (unpartitioned) set, with preserved robustness. 
Patients within the same subset should react similarly to drug infusions and surgical disturbances. Then, how can this similarity be quantified? In this thesis, different methods of comparing patient models and clustering them into subsets have been tested. Due to combinatorial complexity, not all possible partitions of the patient model set are possible to evaluate. Instead, some clustering heuristics will be proposed and compared.

All partitions were done in two subsets except for partitioning based on delay, which also was partitioned into three subsets. The reason for partitioning in three subsets was to see if there would be any improvement in implementing three subsets instead of two. Partitioning in two subsets was made with median cut, meaning that all patient models with values above the median belongs to a certain subset and all values below the median belongs to the other subset. The reason that median was used is to create two subsets of approximate same size. It might of course be favourably to make another division due to the distribution of data. Since it was unclear where the limit should be drawn, median cut was implemented. For $n$ subsets, the limits for separation between subsets is percentiles, creating subsets of about the same size. For $n$ subsets, the percentiles are $\frac{100}{n} / \frac{2 \cdot 100}{n} / \ldots / \frac{(n-1) \cdot 100}{n}$.

\subsection{Partitioning based on physical characteristics}

One possibility is to base the partitioning on demographic data such as age, gender, weight and height. The advantage of partition on these characteristics is that they are known beforehand.

Partition on gender has been done for one subset with men and one with women. The other partitions based on physical characteristics have been made using median cut for partition into two subsets.

\subsection{Partitioning based on delay}

Since a long model time delay $T_{d}$ limits the performance of the controller and makes it more conservative, it is intuitive to partition the patient models depending on the delay. Two subsets were created using median cut on delay and three subsets are created using cut on suitable percentiles, see Section 3.1. The reason for choosing partitioning in three subsets based on delay (and not the other methods) is that this method was expected to result in the best attenuation of surgical disturbances. The data distribution of delay in the two model sets are shown in Figure 3.1 and 3.2. As seen in the figures, the distribution of delays shows of no clear division an therefore it was not clear of how the partitioning should be made.

If a controller should be chosen depending on the delay, suitable settings can not be decided before the anesthesia. When a new patient is to be anesthetized, a conservative controller is chosen in absence of information. The actual delay must 


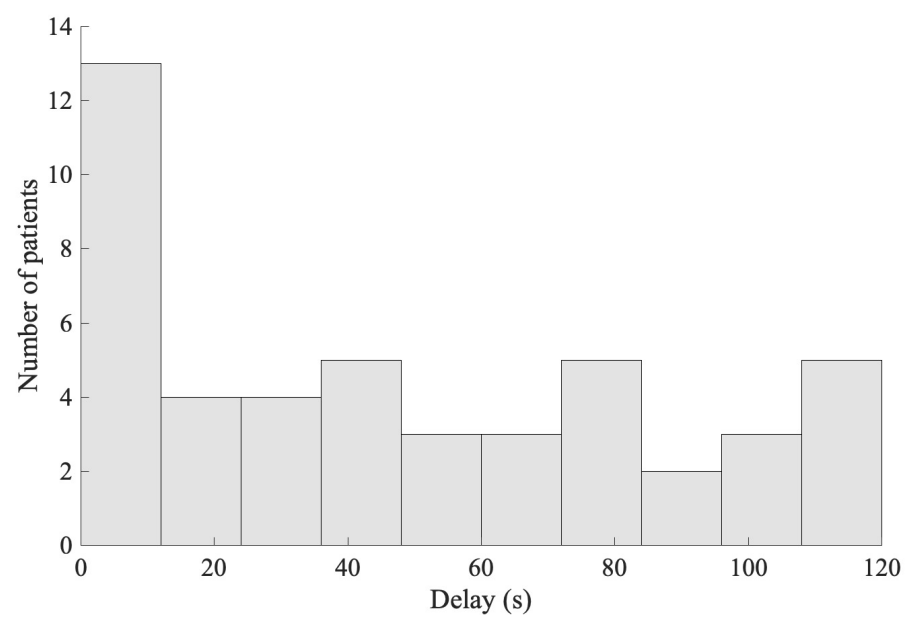

Figure 3.1: Distribution of delays $T_{d}$ from provided children model set.

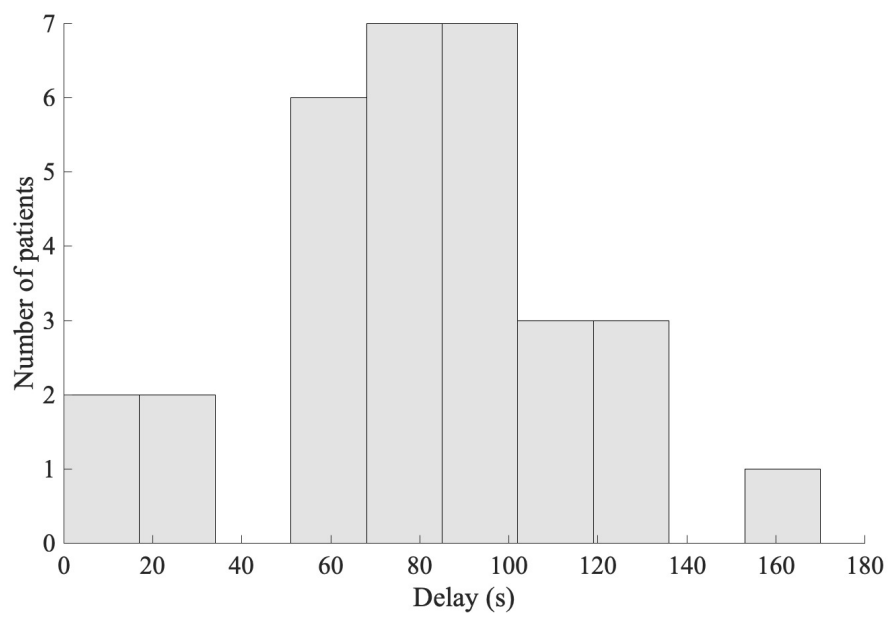

Figure 3.2: Distribution of delays $T_{d}$ from provided adult model set. 




Figure 3.3: The proposed method for estimating delay from induction data. The $\mathrm{DOH}$ is marked in blue, the initial value $E_{0}$ is marked as red dashed line and the limit is marked with a red line. The estimated delay is marked with a gray vertical line.

then be estimated online. Estimation can be done during the induction phase when there is no surgical stimulation and a more exciting control signal. When the delay has been estimated, there can be a change to a more suitable controller.

In the following section a proposed method for determining the delay online using induction phase data is proposed. The method has been developed with online use in mind. Classification on delay has been made using simulations of the induction phases and on the clinical induction phase data from which the models in [van Heusden et al., 2013] and [van Heusden et al., 2017] were obtained. The simulations were made both with and without noise, modeling measurement noise as described in Section 3.5. $E_{0}$ was estimated as the mean value from the first 30 seconds of DOH data, before any infusion. Then the delay is estimated as time from infusion until the DOH drops below the level $E_{0}-\delta$ for a certain time $t$, where $\delta$ and $t$ need to be decided. An image of the proposed method used on simulated data is shown in Figure 3.3.

The parameters $t$ and $\delta$ were determined from simulations with and without noise on the children model set using the nominal controller. Later on the same parameters were used on the adult model set. The resulting parameters to the proposed method was $t=10$ seconds and $\delta=1.5$. This method was applied on simulated and clinical induction data.

Depending on the estimated delay, a patient model was placed in the subset for 
short or long delay. The limit for being placed in a subset with short or long delay was chosen as the median of the given delays for children and adults.

\subsection{Partitioning based on on $v$-gap}

\section{The $v$-gap metric}

One way of partitioning the patient models into two subsets is to take more information into account. A method that is regularly used for comparing transfer functions is the $v$-gap, described in [Vinnicombe, 2001]. The $v$-gap is a metric, such that processes that behave similarly under (unitary) negative feedback, have a small $v$-gap. That is, if $\delta_{v}\left(P_{1}, P_{2}\right)$ is small then any satisfactory controller for $P_{1}$ will also be satisfactory for $P_{2}$ [Vinnicombe, 2001]. This method is more purposeful than comparing delays because the resulting closed-loop system is taken into account. A drawback is that a dynamic patient models needs to be identified to calculate the $v$-gap.

The $v$-gap metric can be used as a comparison between two stable LTI systems, here represented through their transfer function. Transfer functions can be visualized as Nyquist curves in the complex plane.

To compute the $v$-gap, the Nyquist curves of the corresponding systems are projected onto the Riemann sphere. The Riemann sphere is a sphere of unit diameter, tangenting the complex plane at origo. The Euclidean distance between two projected points of the Nyquist curves onto the sphere is called the chordal distance, see Figure 3.4 [Vinnicombe, 2001].

The chordal distance $d_{c}$ in the scalar case $\left(z_{1}, z_{2} \in \mathbb{C}\right)$ is defined as

$$
d_{c}\left(z_{1}, z_{2}\right)=\frac{\left|z_{1}-z_{2}\right|}{\sqrt{1-z_{1}^{*} z_{1}} \sqrt{1-z_{2}^{*} z_{2}}},
$$

where $z^{*}$ denotes the complex conjugate of $z$. The chordal distance between two

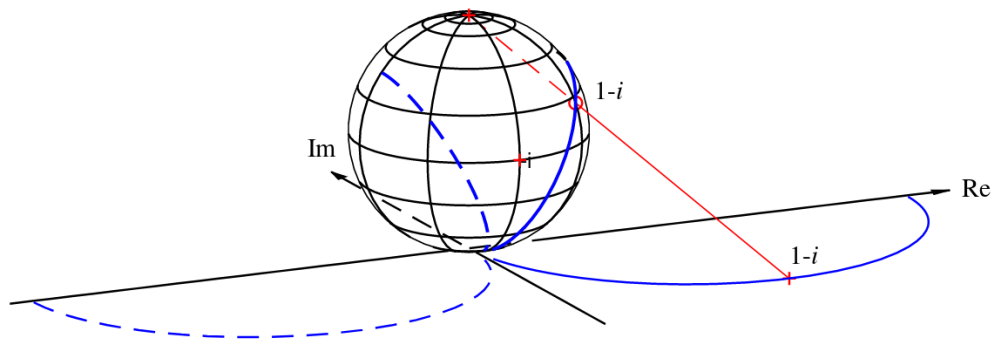

Figure 3.4: Projection of transfer functions onto the Riemann sphere, retrieved from [Åström and Murray, 2008] with permission from Karl Johan Åström. 
transfer functions $P_{1}$ and $P_{2}$ can be expressed as

$$
\kappa\left(P_{1}(j \omega), P_{2}(j \omega)\right)=d_{c}\left(P_{1}, P_{2}\right)=\frac{\left|P_{2}(j \omega)-P_{1}(j \omega)\right|}{\sqrt{1+\left|P_{1}(j \omega)\right|^{2}} \sqrt{1+\left|P_{2}(j \omega)\right|^{2}}},
$$

where $\kappa$ is the point-wise gap between the two systems at frequency $\omega$. From this we can define the $v$-gap metric as the maximum distance between the Nyquist curves for all frequencies [Vinnicombe, 2001]

$$
\delta_{v}= \begin{cases}\sup \kappa\left(P_{1}(j \omega), P_{2}(j \omega)\right), & \text { if winding number condition holds } \\ 1, & \text { otherwise }\end{cases}
$$

where the winding number condition is given by

$$
\delta_{v}<1 \Leftrightarrow\left\{\begin{array}{l}
\operatorname{det}\left(I+P_{2}^{*} P_{1}\right)(j \omega) \neq 0 \\
\operatorname{wno} \operatorname{det}\left(I+P_{2}^{*} P_{1}\right)+\eta\left(P_{1}\right)-\eta\left(P_{2}\right)=0,
\end{array} \quad \forall \omega,\right.
$$

where wno denotes the winding number. The winding number can be stated as $\mathrm{wno}(\mathrm{g})=\frac{1}{2 \pi} \times$ the net increase in the argument of $g(j \omega)$ as $\omega$ decreases from $+\infty$ to $-\infty$ [Vinnicombe, 2001]. The number of open right half plane poles of the plant $P$ is named $\eta(P)$. In the models used in this thesis, there are no poles in the right half plane. For all of the anesthesia models, the winding number condition holds.

The $v$-gap can be calculated for each pair of patient models, $P_{1}$ and $P_{2}$, and the result can be represented using the dissimilarity matrix $C$, where $C_{i j}$ is the $v$-gap between patient $i$ and $j$ and $C_{i i}=0$. The affinity/similarity matrix $A$ is therefore $A=I-C$. The affinity matrix is symmetric with values of pairwise similarities, where 0 represents equality and 1 maximal dissimilarity..

\section{Partitioning using the affinity matrix}

From the affinity matrix (or the dissimilarity matrix) one can perform clustering to determine which patient models should belong to the same subset, i.e. have low value of the $v$-gap. There are different methods to perform such clustering, but since we do not have the exact coordinates but only pairwise distances, it leaves us with fewer alternatives. Note that the pairwise distances are not Euclidean and that the space is not known, which further complicates things.

The most common methods to determine the clusters from an affinity matrix are hierarchical and spectral clustering. Different clustering methods should give similar result if the data contains natural clusters [Bryan, 2004].

\section{Hierarchical clustering}

Hierarchical clustering considers pairwise distances. It does not require a predefined number of clusters as input. The resulting clusters are typically visualized in a dendrogram, see Figure 3.5. Elements are merged together and a merge is shown 


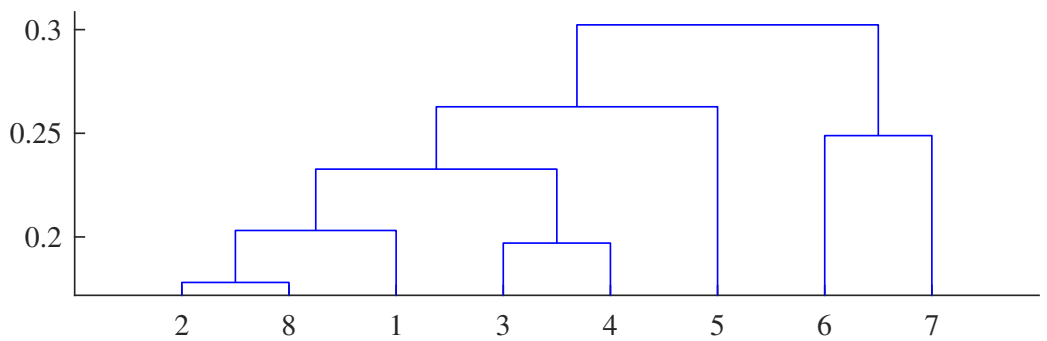

Figure 3.5: A dendrogram showing the result of hierarchical clustering.

as a horizontal line. The x-axis shows the number of elements to be clustered and the y-axis displays the distance between two clusters that are connected, called the linkage distance. The described method is an agglomerative method, or "bottom up", meaning that clusters are created pairwise and then merged as they move up in hierarchy and create larger clusters. This means that the method is invariant on starting point.

How the elements in the data set should be connected are determined by a linkage function. The linkage function uses the distance information from the $v$-gap calculation and links pairs that are close (similar) into clusters made up of two elements. Then, these binary clusters are linked to each other to create bigger clusters until the entire data set are linked together in a hierarchical cluster tree, as shown in Figure 3.5.

The most common linkage methods are single linkage, complete linkage, average distance. If we had Euclidean distances, other methods such as Ward's method [Ward, 1963] could be an alternative. Single linkage, complete linkage and average distance are illustrated in Figure 3.6. Single linkage is sometimes also called nearest neighbour. Clusters are merged together so that the similarity between two clusters are determined by the smallest distance between elements in the two clusters. The single linkage method can be expressed as [Manning et al., 2008]

$$
l(r, s)=\min \operatorname{dist}\left(x_{r i}, x_{s j}\right),
$$

where $x_{r i}$ is the $i^{\text {th }}$ element in cluster $r$ and corresponding for $s$ and $j$.

Complete linkage merges two clusters together so that their similarity are determined by the largest distance between elements in the clusters. The method can be expressed as

$$
l(r, s)=\max \operatorname{dist}\left(x_{r i}, x_{s j}\right),
$$

Average linkage is a combination of the two methods above, expressed as

$$
l(r, s)=\frac{1}{n_{r} n_{s}} \sum_{i=1}^{n_{r}} \sum_{j=1}^{n_{s}} \operatorname{dist}\left(x_{r i}, x_{s j}\right)
$$

where $n_{r}$ is the number of elements in cluster $r$ and $n_{s}$ is the number of element in cluster $s$. 




(a) Single link

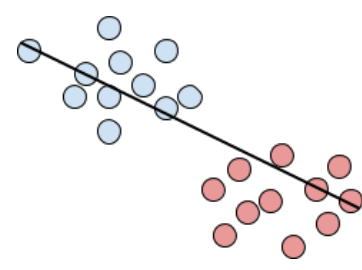

(b) Complete link

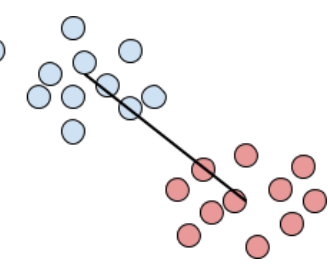

(c) Average link

Figure 3.6: Linkage methods in hierarchical clustering.

Depending on the choice of linkage method, resulting clusters may be different in shape. For example, single linkage has a tendency to create clusters where nearby elements of the same cluster have short distances between them, but elements of opposite ends of a cluster may be far from each other. This is taken care of with the complete linkage method. The drawback with the latter method is instead that it is more sensitive to outliers [Manning et al., 2008]. The average distance method is a combination of single and complete linkage. In this thesis, the average distance linkage method is used, since no information is known about the shape of the clusters.

\section{Spectral clustering}

The general idea of spectral clustering is to look at eigenvalues and eigenvectors of the similarity matrix. Two algorithms for spectral clustering are used in this thesis. We have here labeled the algorithms Shi and $\mathrm{Ng}$, after the first authors of [Shi and Malik, 2000] and [Ng et al., 2001], in which they were introduced.

The Shi algorithm make use of the unnormalized graph Laplacian matrix $L$, defined as [Shi and Malik, 2000]

$$
L=D-A,
$$

where $A$ is the affinity matrix and $D$ is the degree matrix. The diagonal matrix $D$ is defined as a matrix where the $(i, i)$-element is the $i^{\text {th }}$ row sum of $A$.

The algorithm partitions the graph by looking at the eigenvector corresponding to the second smallest eigenvalue. The partition splits the eigenvector in two (or $k$ ) clusters using a splitting point. Choosing an appropriate splitting point can be done in different ways and one proposed choice is to use the splitting point 0 , see [Shi and Malik, 2000]. This will give us the final partition.

The $\mathrm{Ng}$ algorithm uses the normalized graph Laplacian $L_{\text {sym }}$, defined as $[\mathrm{Ng}$ et al., 2001]

$$
L_{\text {sym }}=D^{-1 / 2} L D^{1 / 2},
$$

The algorithm takes $k$ eigenvectors $u_{1}, \ldots, u_{k}$ corresponding to the largest eigenvalues and constructs a matrix $U$ with the eigenvectors as columns. Then this matrix 
is normalized so that each row of $U$ has unit length

$$
\bar{U}_{i j}=U_{i j} /\left(\sum_{k} U_{i k}^{2}\right)^{1 / 2} .
$$

After this, a standard clustering method is used on $\bar{U}$ assuming $k$ clusters to get the final result. One can for example use K-means clustering [Ng et al., 2001]. Kmeans clustering can be used since the data has been projected to a lower dimension and is therefore more easily separable than before. K-means clustering cannot be used directly since the actual positions of the coordinates are not known.

Ready-made functions for hierarchical clustering in MATLAB have been used in this thesis. They are called 'linkage' and 'cluster'. The methods for clustering using Shi and $\mathrm{Ng}$ methods was implemented using the pseudo-code in [von Luxburg, 2007]. In the Shi method, a limit must be chosen for the eigenvalues to make a partition. This value is chosen as the median value of the eigenvalues to create subsets of equal size.

\subsection{Controller structure}

For simulations in this thesis, the structure given in Figure 3.7 has been used. The patient is modeled with a PKPD model, described in Chapter 2. The DOH is controlled using a controller. The controller compares the reference value, $r$, and the measurement value, $y$. The reference value $r$ is the desired depth of hypnosis and $y$ is the measured clinical effect, the $\mathrm{WAV}_{\mathrm{CNS}}$ index. Input $u$ is the infusion rate of the drug, scaled with patient weight and given in $\mathrm{ml} / \mathrm{kg} / \mathrm{h}$ or $\mathrm{mg} / \mathrm{kg} / \mathrm{min}$.

The signal that controls the process is called the control signal, $u$. One of the most common controllers is the PID controller, which is the controller used in this thesis. Input to the controller is the control error $e$ from which the controller determines a control signal $u$. The error $e$ is defined as $e=r-y_{\text {filt }}$ where $y_{\text {filt }}$ is the filtered output. The PID controller consists of three parts, one proportional part (P),

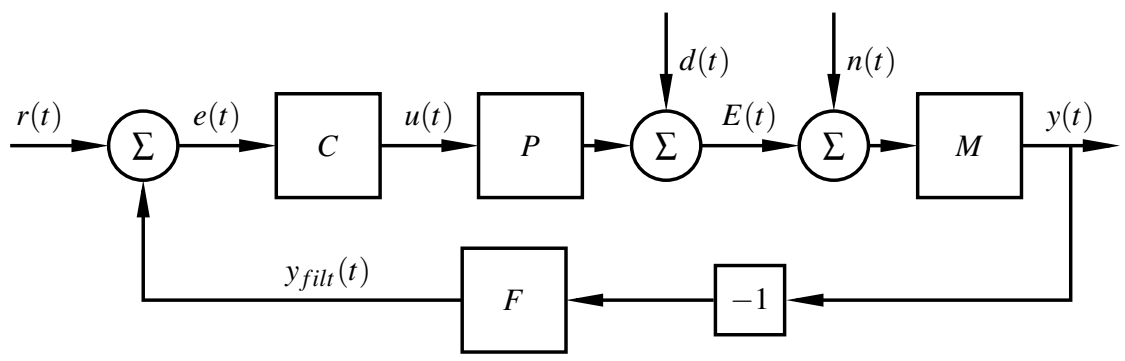

Figure 3.7: Closed-loop control of anesthesia with controller $C$, PKPD model $(P)$, monitor $M$, filter $F$, surgical disturbance $d$ and measurement noise $n$. 
one integrating part (I) and one derivative part (D). The standard form of the PID controller, taken from [Åström and Hägglund, 2006], is

$$
u=K\left(e+\frac{1}{T_{i}} \int e(t) d t+T_{d} \frac{d e}{d t}\right),
$$

where $K$ is the proportional gain, $T_{i}$ is the integral gain and $T_{d}$ is the derivative gain. The PID controller is often expressed in the Laplace domain, as

$$
U(s)=K\left(1+\frac{1}{s T_{i}}+T_{d} s\right) E(s),
$$

Values of $K, T_{i}$ and $T_{d}$ must be chosen carefully to achieve performance and robustness. The parameterization used in this thesis is the parallel form, chosen due to its linearity in the controller parameters,

$$
U(s)=\left(k_{p}+\frac{k_{i}}{s}+k_{d} s\right) E(s),
$$

so that $k_{p}=K, k_{i}=k_{p} / T_{i}$ and $k_{d}=k_{p} T_{d}$. Input $e(t)$ to the controller is converted to the interval $(1,0)$.

Previously used controllers for the children and adult models are denoted nominal controllers. The nominal controller for children used in this thesis was derived and evaluated in [van Heusden et al., 2014]. Its parameters are:

$$
k_{p}=1.1 \mathrm{mg} / \mathrm{kg} / \mathrm{min} / \mathrm{WAV}_{\mathrm{CNS}}\left(\text { normalized) }, \quad k_{i}=0.0061 \mathrm{~s}, \quad k_{d}=66 \mathrm{~s},\right.
$$

and the corresponding controller for adults [Dumont et al., 2011] is

$$
k_{p}=0.81 \mathrm{mg} / \mathrm{kg} / \mathrm{min} / \mathrm{WAV}_{\mathrm{CNS}}\left(\text { normalized) }, \quad k_{i}=0.0055 \mathrm{~s}, \quad k_{d}=45 \mathrm{~s} .\right.
$$

The clinical effect $E(t)$ is measured with the NeuroSense NS-701 monitor. The dynamics are well defined as [Zikov et al., 2006]

$$
M(s)=\frac{1}{(8 s+1)^{2}},
$$

The resulting DOH is affected by measurement noise $n$. High frequency measurement noise are attenuated using a second order filter [van Heusden et al., 2014]

$$
F(s)=\frac{1}{\left(T_{f} s+1\right)^{2}},
$$

where $T_{f}=15 \mathrm{~s}$ is the filter constant.

Surgical disturbances are denoted $d$ in Figure 3.7, and modeled as steps. They increase the depth of hypnosis. Further explanations and modeling of surgical disturbances can be found in [Struys et al., 2004]. The measurements are corrupted by 
noise, denoted $n$, which has been identified to be white with a standard deviation of $0.09 \mathrm{WAV}_{\mathrm{CNS}}$ units, identified in [Soltesz et al., 2012].

In this setup, the surgical disturbances affect the signal before entering the monitor. From this, we can set up the sensitivity function as the transfer function from $d$ to $E$. The sensitivity function is

$$
S(s)=\frac{1}{1+P(s) C(s) F(s) M(s)},
$$

where $P(s)$ is the patient model, $C(s)$ is the controller, $F(s)$ is the filter and $M(s)$ is the monitor.

\subsection{Controller synthesis}

This section is a contribution from José Manuel González Cava, who is preparing a manuscript on controller synthesis for patient model sets of the type resulting from partitioning in this thesis. The section describes the PID controller and measurement filter optimization, which has been used to obtain controllers for the model set, each considered subset, and individual patient models.

The approach has been to maximize some measure of performance, while fulfilling constraints, which provide robustness guarantees. This is accomplished by limiting the $\mathscr{L}_{2}$ norm of the patient output $y$ resulting from a step disturbance $d$, see Figure 3.7. The transfer function from $d$ to $y$ is the sensitivity function $S$ in (3.18).

To maintain robustness, $\mathscr{H}_{\infty}$ constraints on the sensitivity function $S$ and its complement $T=1-S$ are imposed. Attenuation of measurement noise is governed through an $\mathscr{H}_{2}$ (energy) constraint on $K S$, where $K=F C$, the transfer function from measurement noise $n$ to control signal $u$. Maximum undershoot is limited to maximum $M_{y}=0.1$ of the amplitude of the disturbance. The constraint levels of (3.19) were chosen as those corresponding to previously clinically evaluated controllers (3.14) [van Heusden et al., 2013] and (3.15) [van Heusden et al., 2017].

The synthesis objective is formulated to maximize worst case performance over the model set, while maintaining constraints. The optimization problem for the patient model set $\mathscr{P}$ is given by 


$$
\begin{array}{cl}
\min _{K} & \max _{k=1, \ldots, \#(\mathscr{P})}\left\|S_{k} \frac{1}{i \omega}\right\|_{2}^{2} \\
\underset{k \in\{1, \ldots, \#(\mathscr{P})\}}{\text { subject to }} & \left\|S_{k}\right\|_{\infty} \leq M_{s} \\
& \left\|T_{k}\right\|_{\infty} \leq M_{t} \\
& \left\|K S_{k}\right\|_{2} \leq M_{k s} \\
& \mathscr{L}^{-1}\left(S_{k} \frac{1}{i \omega}\right) \geq M_{y} .
\end{array}
$$

The optimization was done in two steps: a global optimization followed by a local one. The two-step approach was considered since the optimization problem in general is non-convex meaning there can be multiple local optima. The global optimization uses the algorithm simulated annealing, originally published in [Kirkpatrick et al., 1983]. To understand the concept of the algorithm, one can compare it with minimizing the energy of a system. When cooling a substance slowly, it will sooner or later come to the lowest energy state. But, if the cooling acts quickly, the substance might not fall into its lowest energy state and be caught in a local minimum, even if it is fully cooled. This comparison was made in [Kirkpatrick et al., 1983] and is one argument for the algorithm. The algorithm is designed to perform well on systems with multiple local optima.

The local optimization is nonlinear and gradient-based, called SLSQP based on exact Jacobian and Hessian computations through dual complex numbers [Kandasamy and Smarandache, 2012]. SLSQP uses gradients to find the minimum where the gradient is zero. The starting point was the result from the global optimization and the method is iterating until a minimum is found [Kraft, 1988].

The optimization algorithm yields the controller parameters $k_{p}, k_{i}, k_{d}$ and the filter coefficient $T_{f}$ together with the resulting costs for each patient model after global and local minimization where the cost is given as the $\mathscr{L}_{2}$ norm described above. 


\section{4}

\section{Results}

The results will primarily be presented though box plots, where the box shows median as a red line, the $25^{\text {th }}$ and $75^{\text {th }}$ percentile in blue, whiskers showing minimum and maximum and outliers plotted individually as red ' + '. Outliers are values greater than $q_{75}+1.5\left(q_{75}-q_{25}\right)$ and values smaller than $q_{25}-1.5\left(q_{75}-q_{25}\right)$ where $q_{25}$ and $q_{75}$ are the $25^{\text {th }}$ and $75^{\text {th }}$ percentile of the data, respectively.

\subsection{Initial controller and best case}

As a first approach before the partitioning, the best and worst case is considered for comparison. Best case denotes the case when the largest possible attenuation of surgical disturbances over the model set is achieved, so that an individual controller is synthesized for each patient. An individual controller for each patient is not possible to implement as explained in Chapter 2, but is used for comparison. In the figures are individual controller for each patient denoted 47 subsets. Worst case denotes the (initial) nominal controllers in (3.14) and (3.15). This is denoted Nominal, 1 set. A synthesized controller over the full model set are denoted Optimal, 1 set.

Costs for children are shown in Figure 4.1 with nominal, optimal and individual controllers. The leftmost box plot displays the costs for the children models using the nominal controller and the box in the middle displays the cost for all children models with the same optimized controller. On the y-axis is the cost calculated as the $\mathscr{L}_{2}$ norm (cost) scaled with the minimum value for the individualized controllers, shown in the rightmost box plot. This scaling has been performed to facilitate comparison and is present in all box plots. Figure 4.2 shows the corresponding boxes for the adult model set. Note that for adults, the costs for the optimal controller on the entire model set are higher than for the nominal one. This is due to that the margins are chosen from the children nominal controller and they are larger than the margins for the adults nominal controller.

Simulated step responses resulting from a change in the reference $r$ associated with Figures 4.1 and 4.2 are shown in Figure 4.3 and 4.4, respectively. The figures 




Figure 4.1: Patient cost distribution for children model set using a nominal controller, an optimal controller for the entire model set and an individual controller for each patient model.



Figure 4.2: Patient cost distribution for adult model set using a nominal controller, an optimal controller for the entire model set and an individual controller for each patient model. 


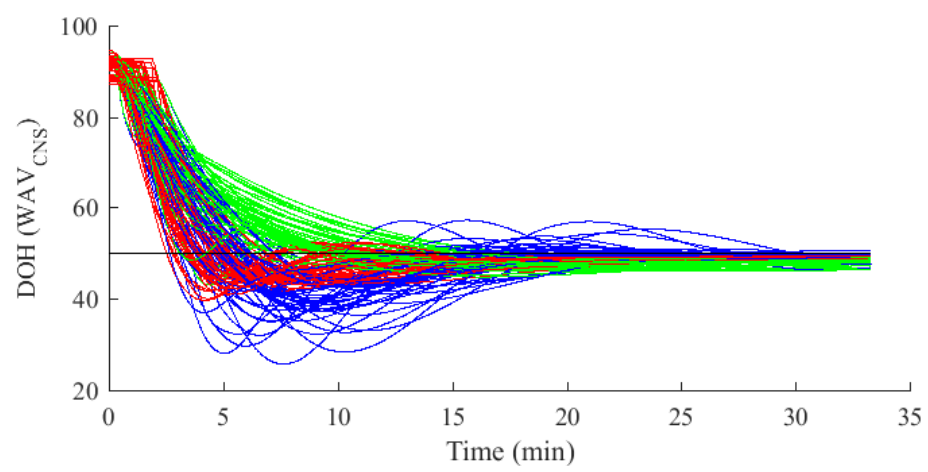

(a) DOH measurement



(b) Propofol infusion rate $u$

Figure 4.3: Simulations comparing step response for children model set using nominal controller (blue), optimal controller (green) and individually calculated controllers for each patient model (red). Set point is in black.

illustrate the $\mathrm{DOH}$ as well as the control signal, the infusion rate. The reference is set to $50 \mathrm{WAV}_{\mathrm{CNS}}$ units in all simulations and marked in black.

As expected, using an individualized controller for each patient gives highly improved step responses compared to using the nominal controller. This improvement can be seen as decreased induction time, smaller undershoot and a shorter settling time. The optimized controller for the entire model set result in longer induction times and smaller drug use than both the nominal controller and using individualized controllers. Step responses for adults have greater overshoot than those for children.

Figures 4.5 and 4.6 both display the costs for the individualized controllers on the $y$-axis. The costs are plotted against delay as well as physical characteristics, described in Table 2.1. Linear regression has been done for each plot, where the $R^{2}$ value is shown in each subcaption. When comparing the cost comparing to different characteristics, it is noticeable that there is a relationship between increased cost 


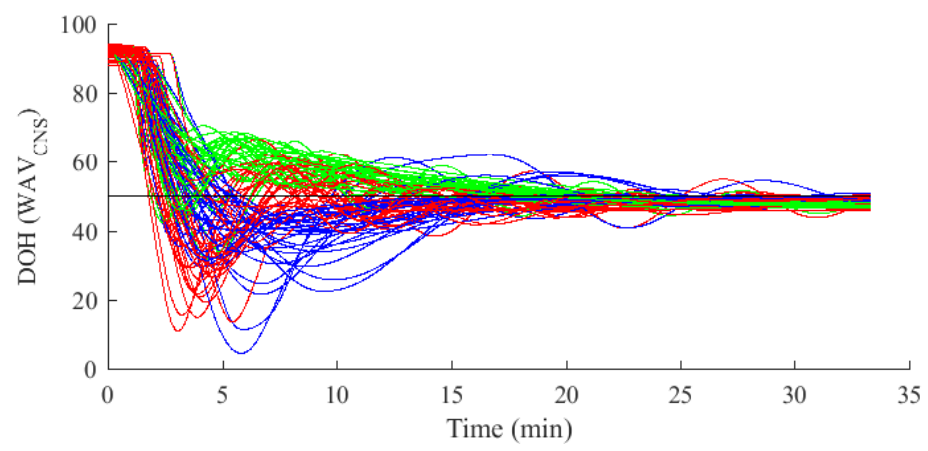

(a) DOH measurement

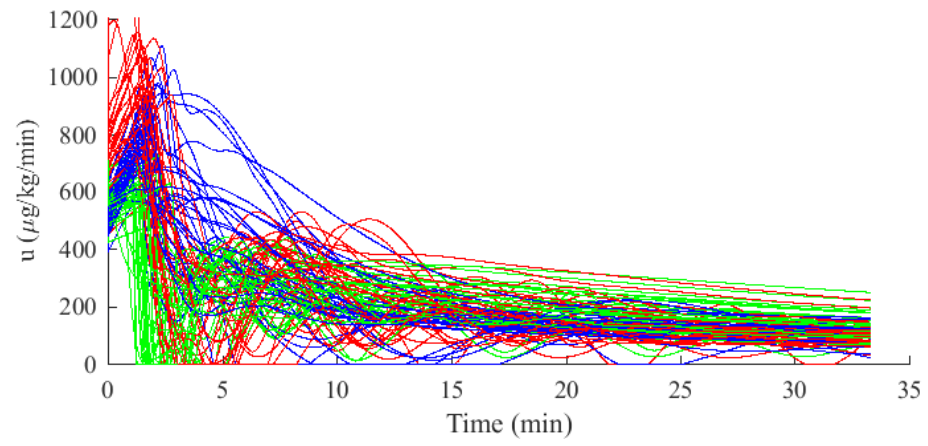

(b) Propofol infusion rate $u$

Figure 4.4: Simulations comparing step response for adult model set using nominal controller (blue), optimal controller (green) and individually calculated controllers for each patient model (red). Set point is in black.

and increased delay. The other attributes shows an almost non-existent correlation to cost except $E_{0}$ that displays a small correlation. 




(a) Delay, $R^{2}=0.7634$

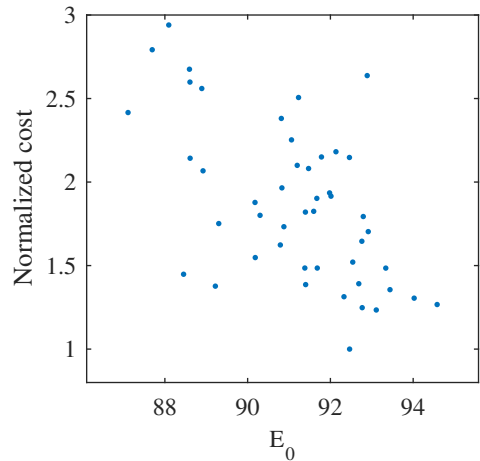

(c) $E_{0}, R^{2}=0.3352$

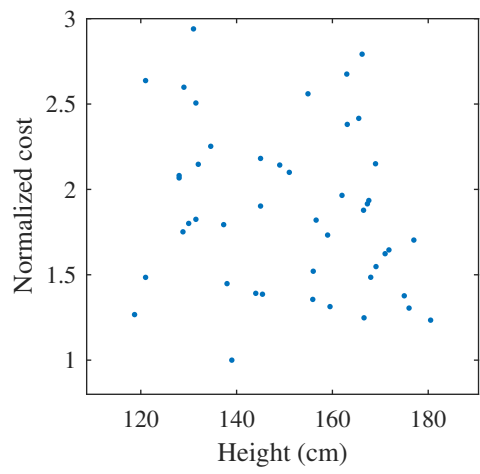

(e) Height, $R^{2}=0.0434$

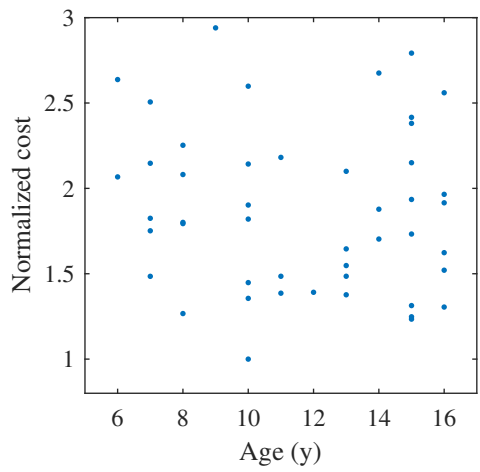

(b) Age, $R^{2}=0.0126$

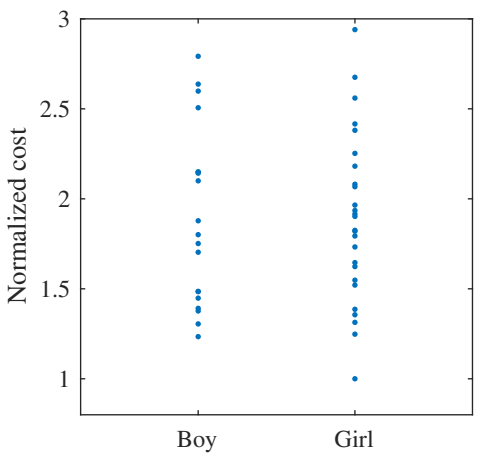

(d) Gender

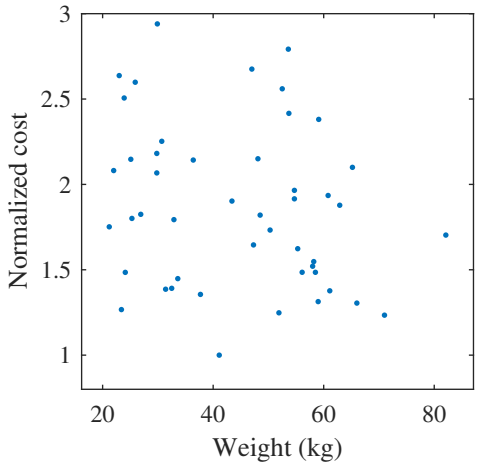

(f) Weight, $R^{2}=0.0500$

Figure 4.5: The ideal case with an individual controller for each patient model where the cost is plotted with different physical attributes and delay. The model set is children 6-16 y. The cost is a measure of the ability to suppress disturbances, where a lower cost means better suppression ability. A linear regression has been made for the data in each plot, where the $R^{2}$ value is shown in the subcaption. 


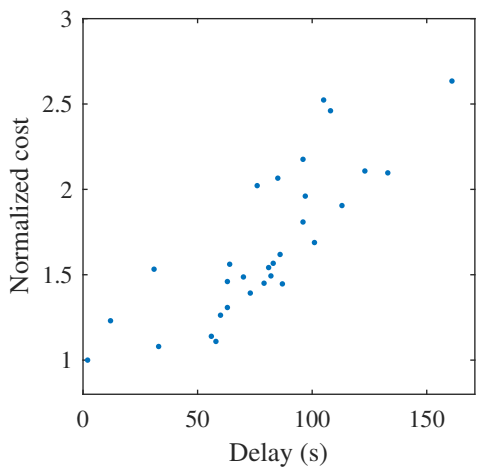

(a) Delay, $R^{2}=0.6078$

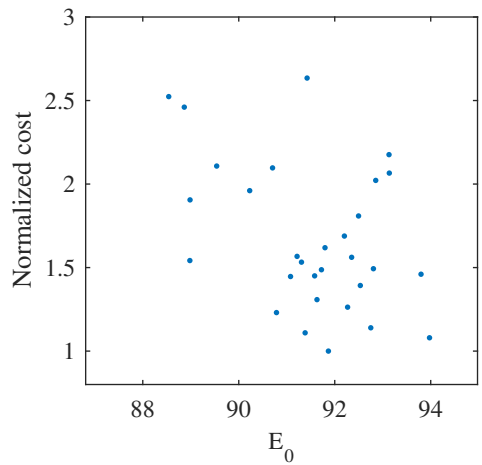

(c) $E_{0}, R^{2}=0.3072$

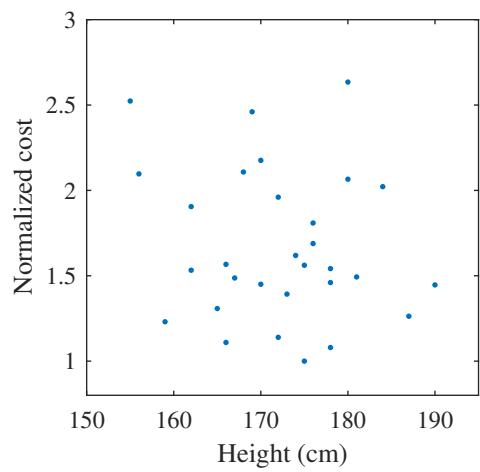

(e) Height, $R^{2}=0.0103$

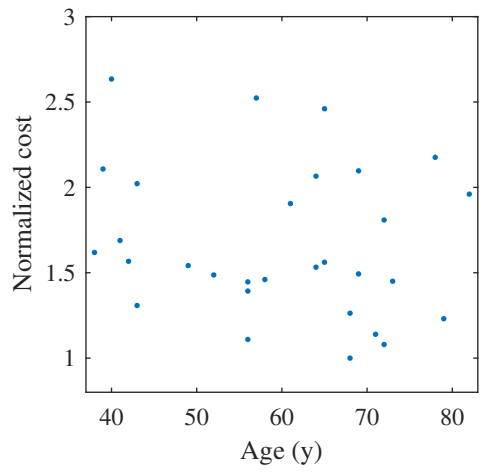

(b) Age, $R^{2}=0.0204$

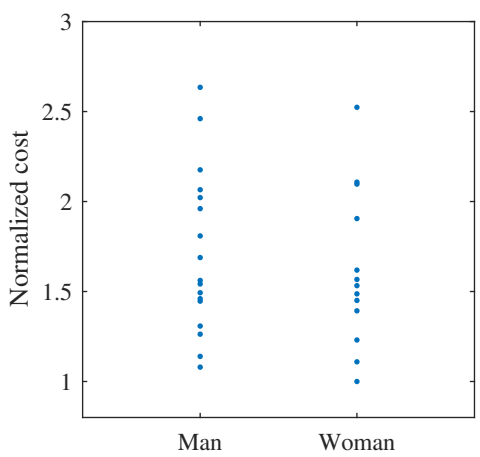

(d) Gender

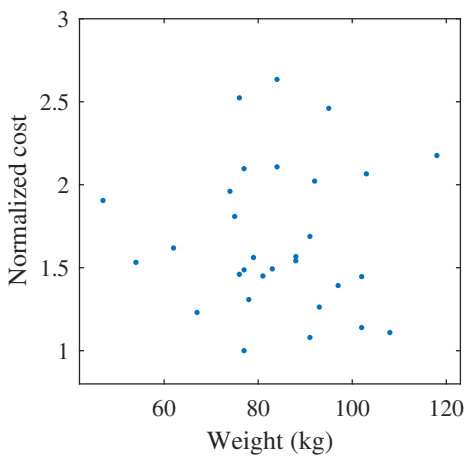

(f) Weight, $R^{2}=0.0028$ 




(g) LBM, $R^{2}=6.67 \cdot 10^{-4}$

Figure 4.6: The ideal case with an individual controller for each patient model where the cost is plotted with different physical attributes and delay. The model set is adults $38-82 \mathrm{y}$. The cost is a measure of the ability to suppress disturbances, where a lower cost means better suppression ability. A linear regression has been made for the data in each plot, where the $R^{2}$ value is shown in the subcaption.

\subsection{Partitioning based on physical characteristics}

Results of partitioning into two subsets on physical characteristics are shown in Figure 4.7 for children and Figure 4.8 for adults. The partitions are made on median, described in Section 3.1. Partition on gender are made on boy/girl and man/woman.

For children, a partition on age or height means a lower cost and are therefore favourable comparing to partitioning based on the other considered demographic characteristics. In the adult case, partitioning based on weight yield the smallest cost. Comparing to the optimal controller for the adult set, partition on gender gives the same worst cost. 


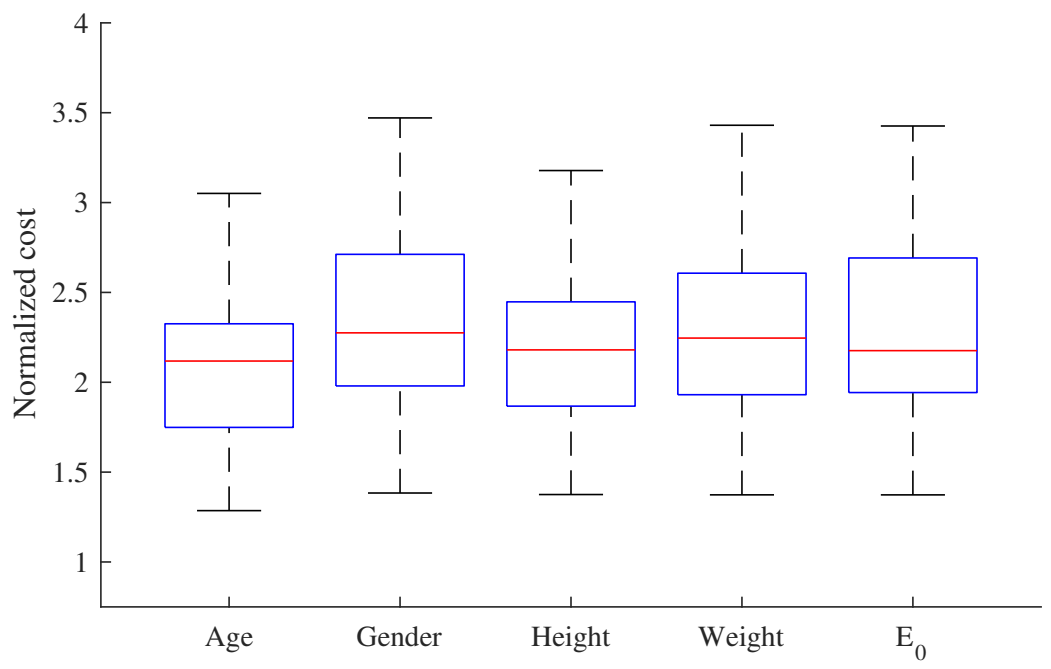

Figure 4.7: Cost for each patient in the children model set using a controller chosen from partitioning. Partitioning is done into two subsets of small/large age, height, weight and $E_{0}$, and between boy and girl.

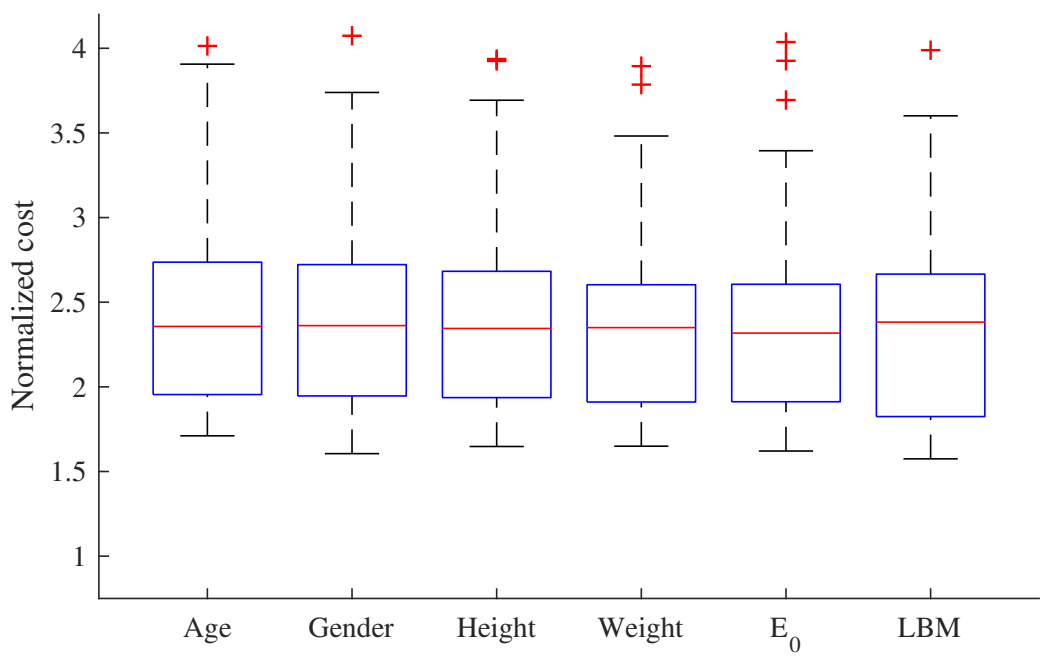

Figure 4.8: Cost for each patient in the adult model set using a controller chosen from partitioning. Partitioning is done into two subsets of small/large age, height, weight and $E_{0}$, and between boy and girl. 


\subsection{Partitioning based on delay}

Partition on delay into two and three subsets compared to one set (explained in Section 4.1) and individual subsets are shown in Figure 4.9 for children and Figure 4.10 for adults. The delay used here for partition is the delay $T_{d}$, introduced in Section 2.1 in the PD models. In the figures, one can see that introducing three subsets instead of two only resulted in a marginal improvement. Also, two subsets resulted in a small average reduction of the cost compared to one set.

The result from estimation of delay using the proposed method are shown in Table 4.1. The proposed method created partitions into short/long delays and these partitions were compared to the partitions into two subsets in Figures 4.9 and 4.10. As seen in the table, the proposed method estimated the delay with good accuracy.

The estimated delays from the proposed method in Section 3.3 differs from values of $T_{d}$ from the provided models. The delays estimated using these two methods are displayed in Figure 4.11 for children and Figure 4.12 and adults.

Simulated step responses resulting from a change in the reference $r$ (see Section 3.5) displaying the result from partitioning based on delay can be seen in Figure 4.13 for children and Figure 4.14 for adults. Here, the patient models have been simulated with their assigned controller according to the classification. The step responses show of a small undershoot and short settling time, even though the induction time seem to have increased slightly compared to the nominal controller in the children set. The average minimum $\mathrm{WAV}_{\mathrm{CNS}}$ index reported in the induction phase was 47 for children and 45 for adults. The adult set showed a tendency to overshoot, where the DOH exceeds the recommended level of 60 for some patient models. Oscillatory behaviour was detected for two patients in the adult model set. The control signal was approximately constant throughout the simulation except for the patients models with oscillatory behaviour.

Bode plots displaying the open-loop transfer functions for linearized discrete models with partitions into two subsets with delay are shown in Figure 4.15 for children model set and Figure 4.16 for adult model set. The partition into two groups on delay for children can easily be spotted in the the phase plot. The division is clear around higher frequencies but is less significant for the adult model set.

Table 4.1: Accuracy for classification on delay with proposed method for two subsets with short/long delay compared to partitions made from delays of the provided models. Classification is done on simulated data without noise, simulated data with noise and clinical data.

\begin{tabular}{cccc}
\hline & $\begin{array}{c}\text { Simulation, } \\
\text { without noise }\end{array}$ & $\begin{array}{c}\text { Simulation, } \\
\text { with noise }\end{array}$ & Clinical data \\
\hline Children & 0.9787 & 0.8723 & 0.8298 \\
Adults & 0.9032 & 0.8387 & 0.7742 \\
\hline
\end{tabular}




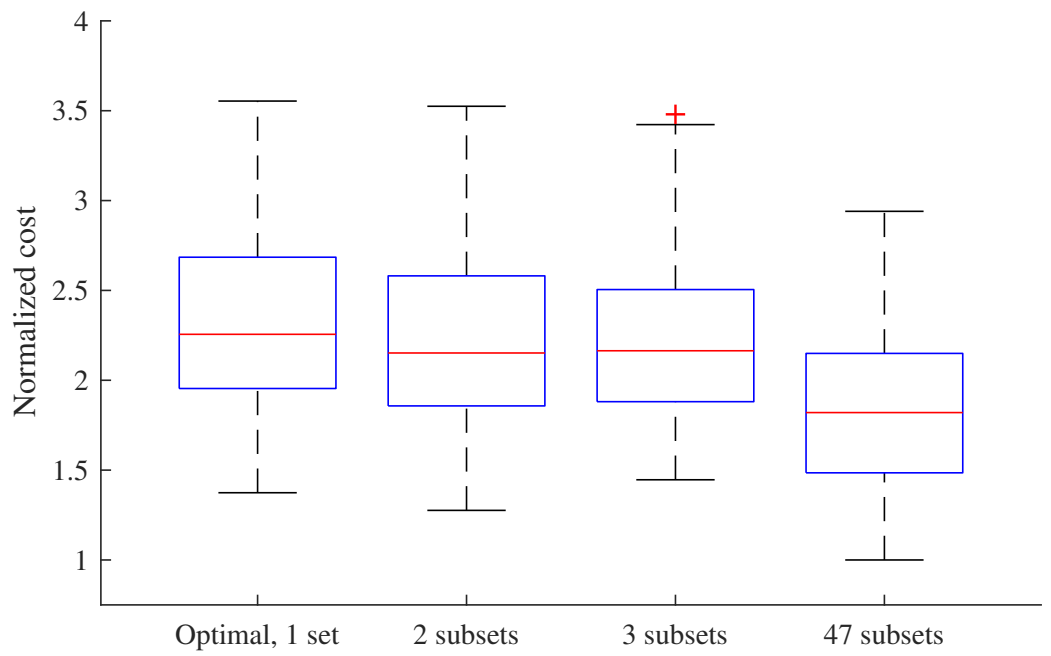

Figure 4.9: Cost for each patient in the children model set using a controller chosen from partitioning. Partitioning is done into two subsets of short/long delay.



Figure 4.10: Cost for each patient in the adult model set using a controller chosen from partitioning. Partitioning is done into two subsets of short/long delay. 




Figure 4.11: Children model set. The estimated and real (from the provided models) delay determined from clinical induction phases, used for classification on delay.

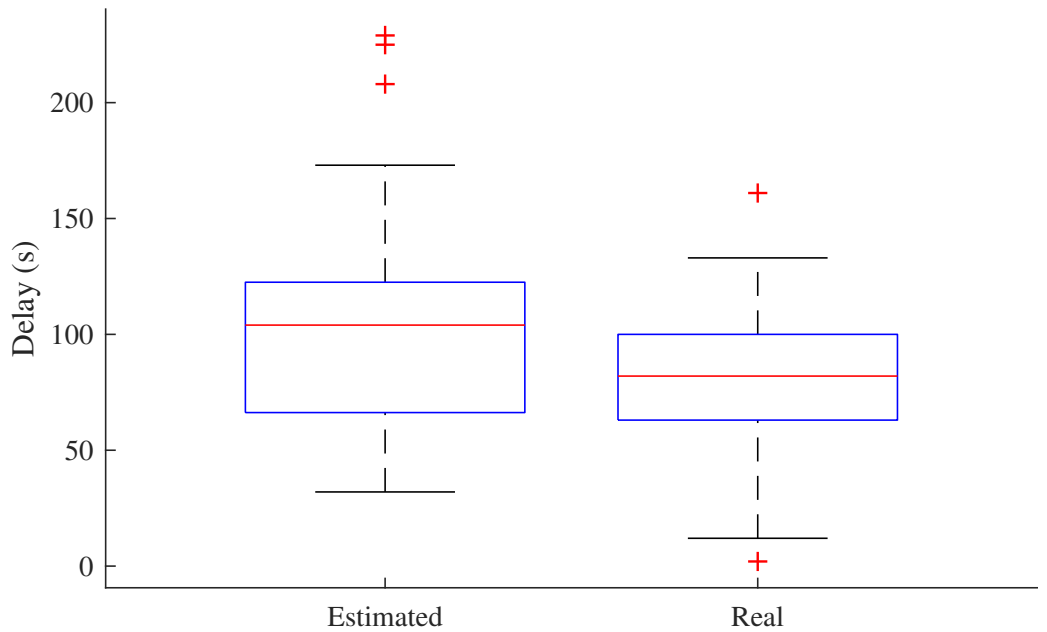

Figure 4.12: Adult model set. The estimated and real (from the provided models) delay determined from clinical induction phases, used for classification on delay. 


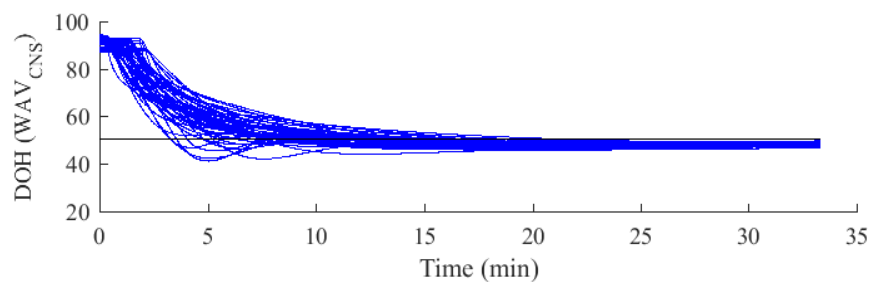

(a) $\mathrm{DOH}$ measurement

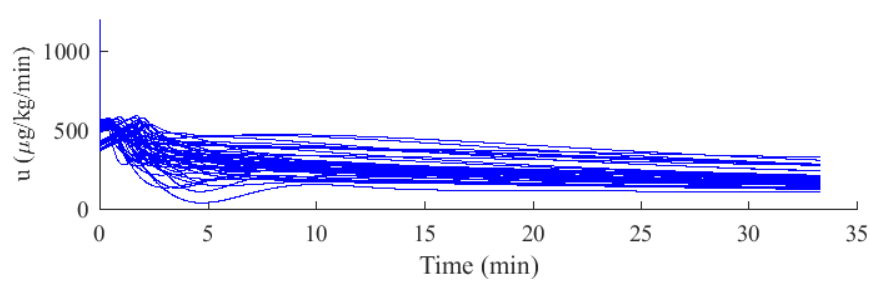

(b) Propofol infusion rate $u$

Figure 4.13: Simulations comparing step response for children model set using nominal controller (blue) and controller chosen according to classification on short/long delay (red) made on clinical induction phases. Set point is in black.

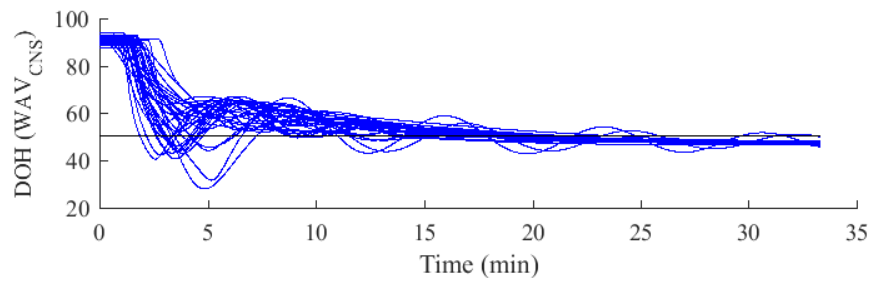

(a) DOH measurement

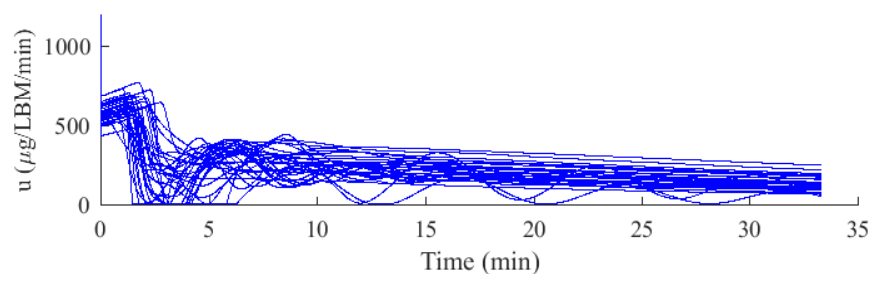

(b) Propofol infusion rate $u$

Figure 4.14: Simulations comparing step response for adult model set using nominal controller (blue) and controller chosen according to classification on short/long delay (red) made on clinical induction phases. Set point is in black. 


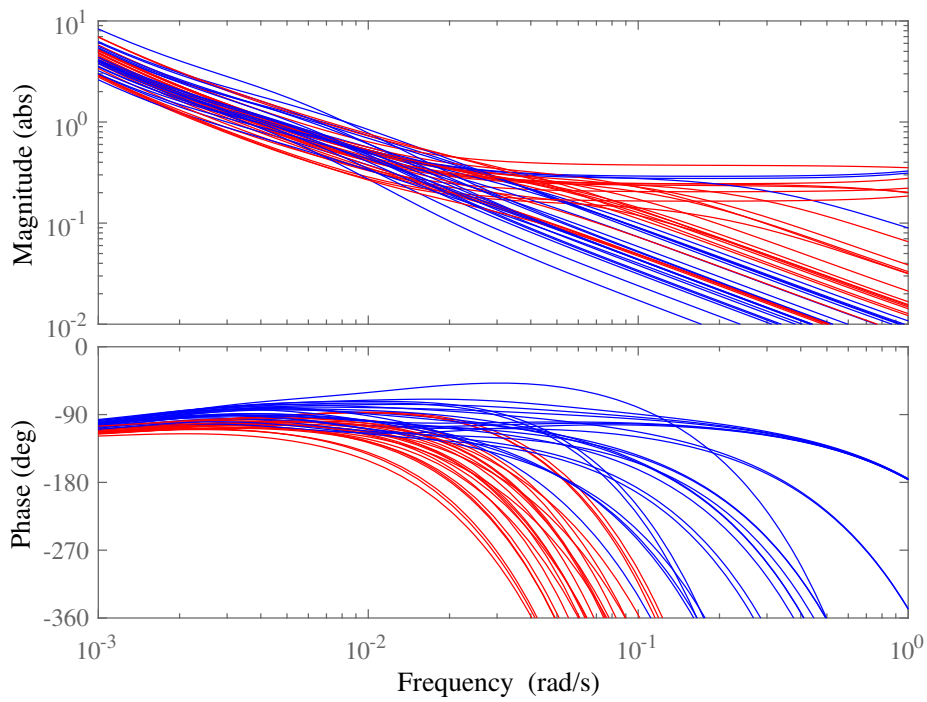

Figure 4.15: Bode plot showing the partition into two subsets (one in blue and one in red) for open-loop transfer function with linearized discrete children models using partition on short/long delay.

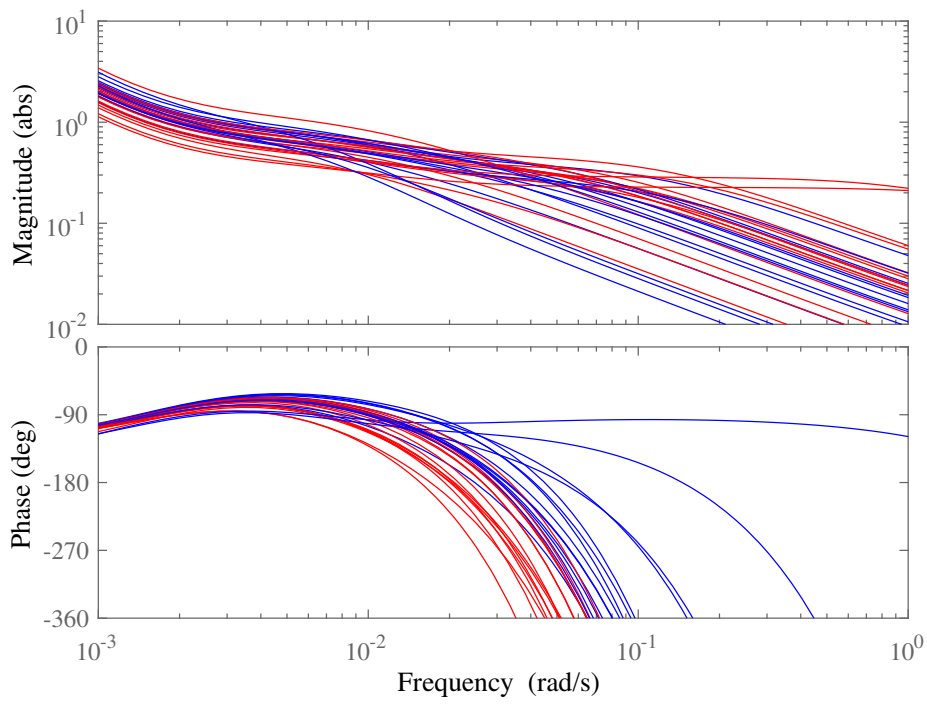

Figure 4.16: Bode plot showing the partition into two subsets (one in blue and one in red) for open-loop transfer function with linearized discrete adult models using partition on short/long delay. 


\subsection{Partitioning based on v-gap}

Dissimilarity matrices obtained from calculating the $v$-gap between all pairs of patient models are shown in Figure 4.17 for children and Figure 4.18 for adults. All pairwise $v$-gaps are shown as a color in the matrix, with patient numbers on the $\mathrm{x}$ and y-axis. The matrix is symmetric so that each pairwise distance is shown two times. Darker blue in the image means more similar and yellow means more dissimilar.

From the dissimilarity matrix, different types of clustering are performed. The dendrograms from the hierarchical clustering are shown in Figures 4.19 and 4.20. The resulting costs using the three clustering methods are shown in Figures 4.21 and 4.22 .

Bode plots displaying the open-loop transfer function for linearized discrete models with partitions into two subsets with $v$-gap and the $\mathrm{Ng}$ method are shown in Figure 4.15 for children model set and Figure 4.16 for adult model set. The partition is clearly identifiable in the bode plot for children for low frequencies, for both gain and phase. For adults, the partition is visible particularly in the phase plot. These bode plots displays that the $v$-gap takes both delay and gain into account in the partitioning.



Figure 4.17: Dissimilarity matrix for 47 patients in the children model set, showing the $v$-gap between all pairs of patient models. Dark blue (zero) means equal and yellow (0.5) means different. 


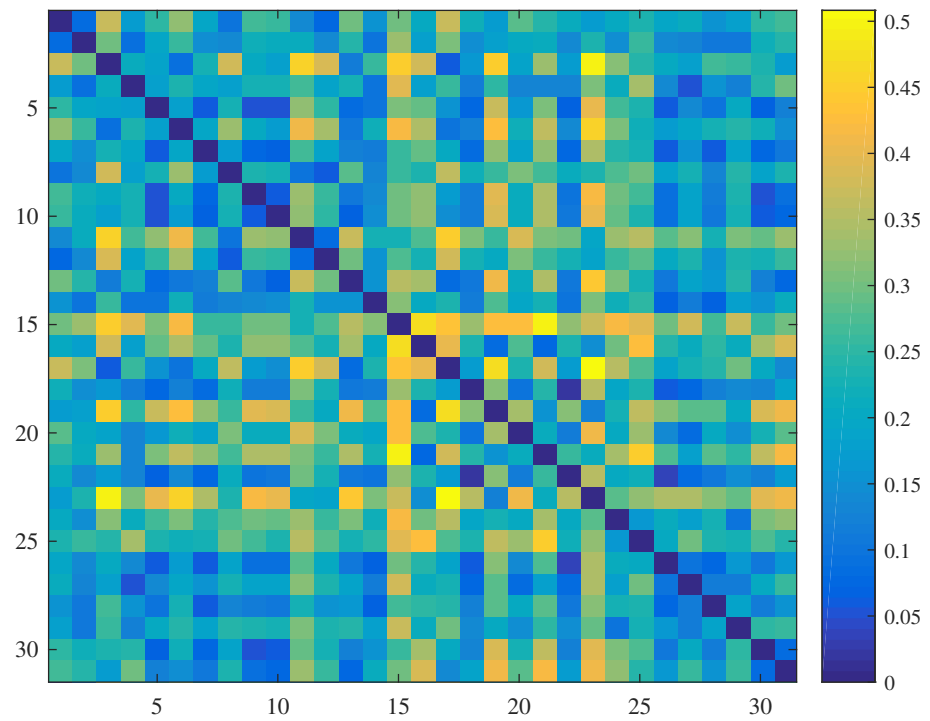

Figure 4.18: Dissimilarity matrix for 31 patients in the adult model set, achieved from $v$-gap between all pairs of patient models. Dark blue (zero) means equal and yellow (0.5) means different.

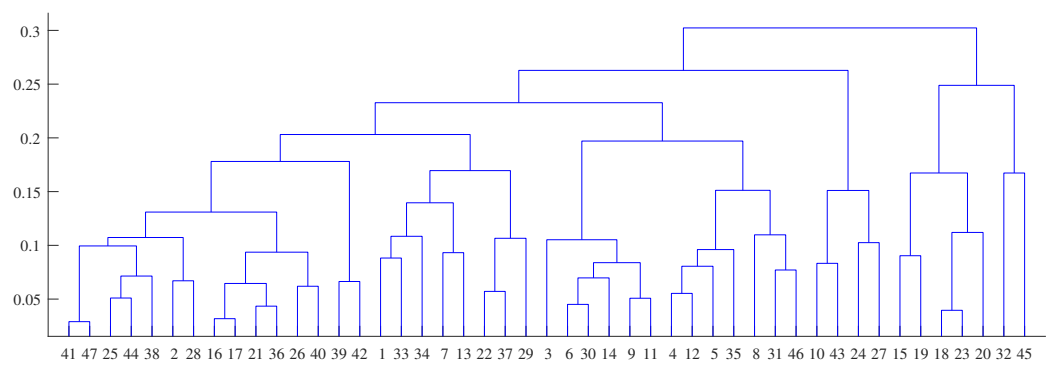

Figure 4.19: Dendrogram obtained through hierarchical clustering performed on the dissimilarity matrix from pairwise $v$-gap between the 47 considered children models. The linkage method was average linkage. 


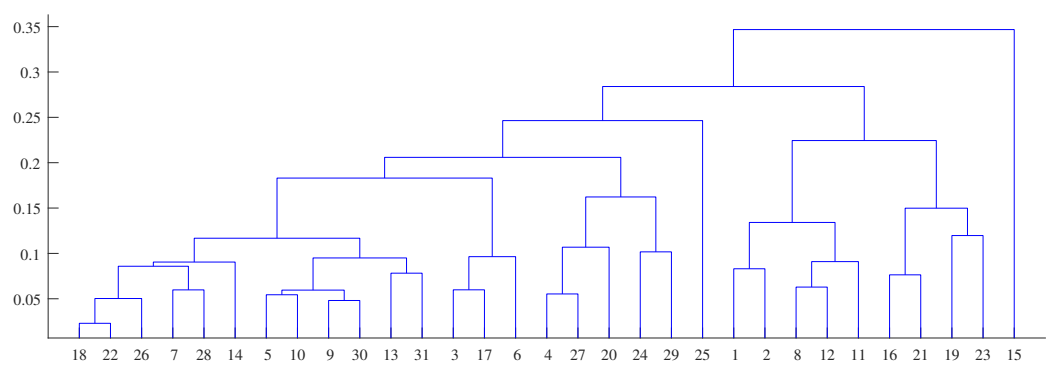

Figure 4.20: Dendrogram obtained through hierarchical clustering performed on the dissimilarity matrix from pairwise $v$-gap between the 31 considered adult models. The linkage method was average linkage.

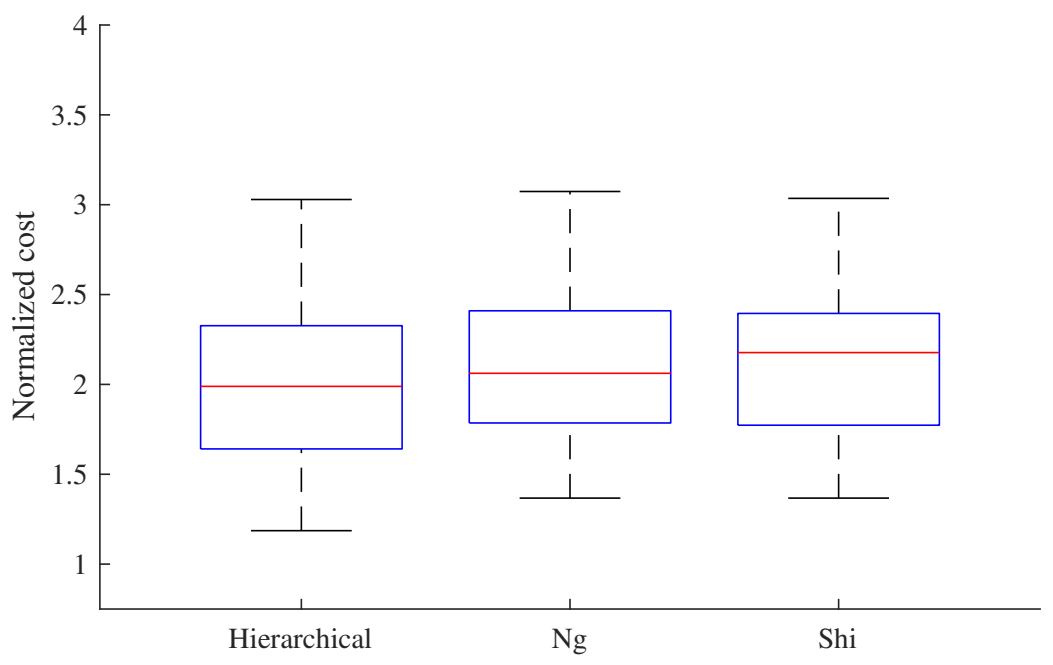

Figure 4.21: Cost for each patient in the children model set using controllers synthetized upon clustering. Partitioning was done into two subsets using $v$-gap and different clustering methods. 


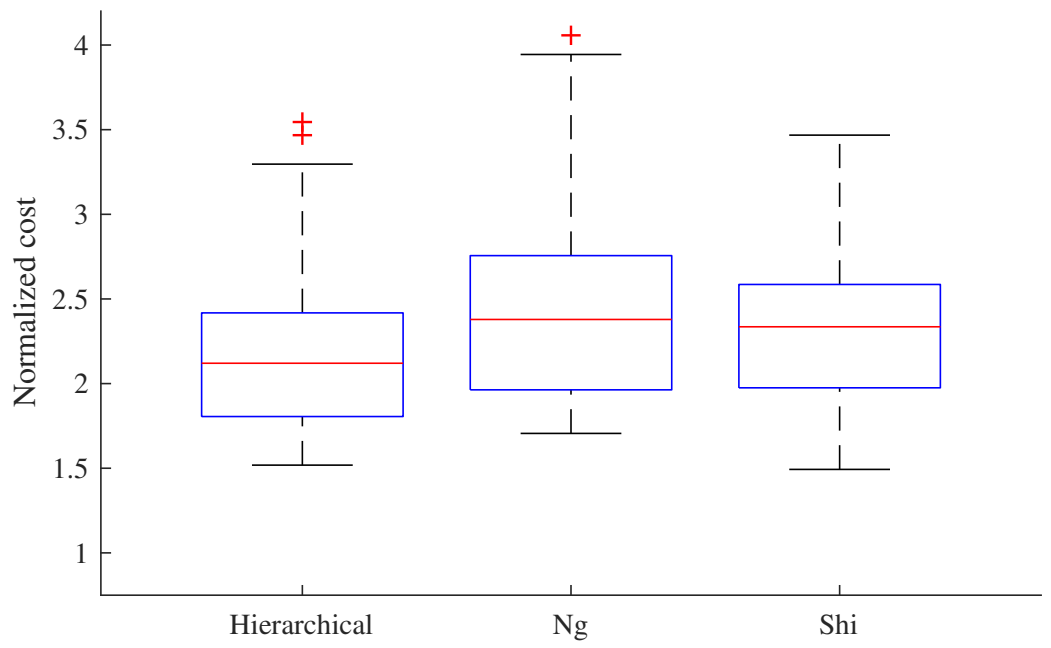

Figure 4.22: Cost for each patient in the adult model set using controllers synthetized upon clustering. Partitioning was done into two subsets using $v$-gap and different clustering methods.

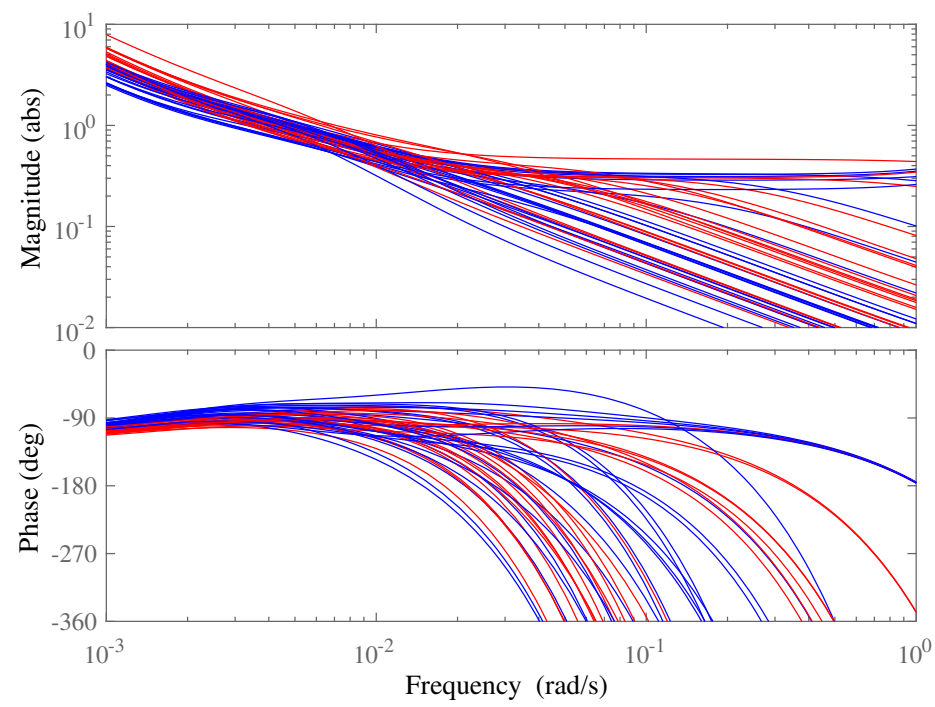

Figure 4.23: Bode plot showing the partition into two subsets (one in blue and one in red) for open-loop transfer function with linearized discrete children models using clustering on $v$-gap. The clustering method was spectral clustering with the $\mathrm{Ng}$ method. 


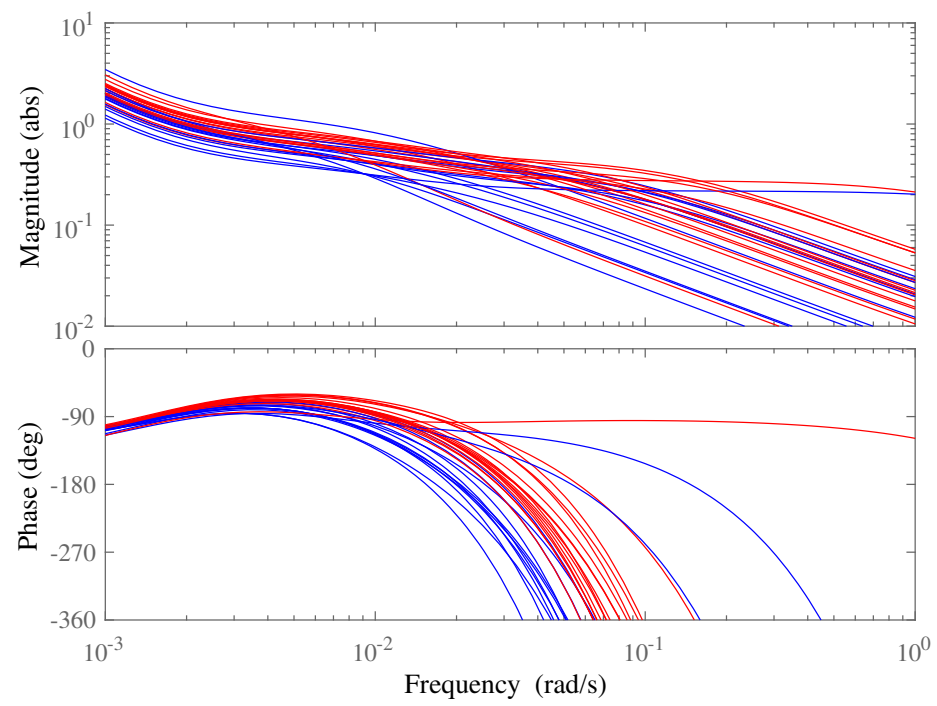

Figure 4.24: Bode plot showing the partition into two subsets (one in blue and one in red) for open-loop transfer function with linearized discrete adult models using clustering on $v$-gap. The clustering method was spectral clustering with the $\mathrm{Ng}$ method. 


\section{5}

\section{Discussion}

Reducing the effects of the inter-patient variability results in safer closed-loop controlled anesthesia. In this thesis, different partitioning methods for minimizing the effects of inter-patient variability have been evaluated.

For our combination of performance measure and robustness constraints, optimization on the entire model set is not sufficient because the resulting controller becomes too conservative. Optimal controllers for each patient is neither possible, due to difficulties to guarantee reliability in identified models. For fewer subsets there are no universal method to find an optimal partitioning, due to the combinatorial complexity. Therefore heuristic methods have been implemented, with all their limitations.

When comparing all of the performed partitions in this work, it is clear that improvement in terms of reduced cost can be achieved by introducing subsets where reduced cost means better attenuation of surgical stimulation disturbances. For partitioning into two subsets, partitioning based on $v$-gap gives the lowest cost for both children and adults. Partitioning based on age for children turned out to be almost as good as partitioning based on $v$-gap. This resulting synthesized controller for children was close in performance to the individually synthesized controllers, with guaranteeed robustness. For adults, the method with the lowest worst cost except for $v$-gap based methods is partitioning based on weight. Partitions into more than two subsets did not show of any significant improvement.

The evaluated partitioning methods have their advantages and disadvantages. The first implemented method was partitioning based on demographic characteristics. Demographic characteristics are simple to measure and are known beforehand and has no significant disadvantage. The second method was partitioning based on delay. The delay is difficult to estimate online and this thesis provides a method for estimating it during induction, which partly resolves this issue since the proposed method overestimates the delay. However, the resulting controllers became less aggressive which is favourable. If the delay is estimated online, a controller must be chosen initially, preferable the controller optimized for the entire model set. After partitioning, a more suitable controller can be switched to. A long time delay was expected to limit the performance of the controller and make it more conservative. 
Therefore, it was expected that partitioning based on delay would result in a low cost. That was not the case and partitioning on delay only gave a small reduction in the worst cost.

The third method handles with partitioning based on $v$-gap. Partitioning based on $v$-gap is not possible to implement in clinical practice as long as a full credible dynamic model cannot be identified during induction. The reason for studying the $v$-gap is to find the best possible partition into two subsets and compare the other partitions with it. If any of the other studied parameters we use for partitioning gives the same result as the $v$-gap, then it can be assumed that this parameter is the most important one in the patient model and the one that we should use for partitioning.

Partitions into two subsets have been made using median cut for partitioning based on delay and physical characteristics. As seen in the histograms in Figure 3.1 and Figure 3.2, the distributions are not clearly separated into two clear subsets. This means that another partition into two subsets could be preferable. This is captured by the clustering with $v$-gap where the clusters differ in size, and the worst cost is low. This leads into analysis of outliers. When looking at the dissimilarity matrices generated by the $v$-gap, one can see some patient models that are standing out. Two examples are patient number 32 and 45 in the children model set in Figure 4.17. These patient models differs a lot from the rest, indicated by yellow in the dissimilarity matrix. They are similar to each other though, and are clustered together in the dendrogram, at the far right in Figure 4.19. In the dendrogram for adults (Figure 4.20), there is one patient model standing out and clustered into its own cluster. Still, the resulting cost from the hierarchical clustering is relatively low which means a good partition.

In this thesis, it has been shown that partitioning into three subsets instead of two only yields a marginal improvement in reduced cost, as seen in Figures 4.9 and 4.10. It is easy to partition into more subsets, but is unnecessary if the profit is marginal.

The controller synthesis method takes the objective of minimizing the sensitivity function when the input is a step while honoring robustness. Both objective and constraints can be chosen differently to receive a controller with other desired properties. The optimization algorithm tries to minimize the worst cost in the entire model set. Another approach could be to minimize the average cost instead. If this approach would be implemented, it will lead to an overall improvement, but may jeapordize the robustness of some individuals.

Simulations display reduced induction time as well as smaller undershoot with controller optimization, both with the entire model set and subsets, compared to the nominal controllers developed in [van Heusden et al., 2014] for children and [van Heusden et al., 2017] for adults. It is unclear if this effect is a coincidence for these specific patient models, or if it is more generic. Regardless, it is a pleasant effect. For adults, two simulations exhibit oscillatory behaviour. It might be relevant to choose other margins for the adult model set to avoid this behaviour.

If no partition would have displayed the same low cost as the $v$-gap, then it 
could mean that the most important parameter is not found and evaluated in this thesis, or that it does not exist a parameter that can be determined a priori or online for partitioning into two subsets for best result. Partitioning based on age for children yields approximately the same result as partitioning based on $v$-gap and it can therefore be concluded that this parameter is important in the children models. For adults, none of the other partitions gave as low cost as partitioning based on $v$-gap and the conclusion that can be drawn from this is that it might not exist a simple parameter upon which the adult models should be partitioned.

\subsection{Limitations}

As in all studies, this project has its limitations. One limitation that has one of the greatest impacts is the limited model set, both for children and adults. It was assumed that this amount of models reflects the population. Also, the adult model set does not contain any adults younger than 38 years. It is of importance to keep in mind that patients that were difficult to model are not part of this study and were excluded in a previous work. Therefore, this study may not reflect the true interpatient variability.

In classification on clinical induction data, it is of importance to remember that limited excitation and a high noise level gives low confidence in the resulting classification. This affects automatic classifiers, such as the proposed method for classification on delay or when a full dynamic model is identified.

\subsection{Future work}

A possible extension of the work presented in this thesis is to implement another classifier that can be used on induction phase data. By using the entire induction phase instead of only the delay, more dynamics (such as induction time) are taken into account. Therefore, it is appropriate to implement a classifier for sequences. One example of classifier that works well for time sequences is a recurrent neural network. The neural network remembers previous data and can be used to predict in which partition a new anesthetized patient should belong, so that similar induction phases will be given the same controller. The classification using a recurrent neural network would be unsupervised and the controller synthesis from this thesis could be implemented as an evaluation of the success of the classification.

In this thesis, partitioning in three subsets were only implemented for partitioning based on delay. It is possible that the improvement from two to three subsets would be more than marginal (as it were for partitioning based on delay) for other partitioning methods. Therefore, it would be interesting to evaluate the partitioning in three subsets for all partitioning methods in this thesis.

It was clear that outlier models affected the resulting costs, particularly in the partitioning based on $v$-gap. If the outliers were excluded in the analysis, the re- 
sulting cost could probably be reduced further. However, by excluding outliers, the model set would lose some of its inter-patient variability which must be considered. 


\section{6}

\section{Conclusion}

Conclusions that can be drawn from the work in this thesis are that the impact of surgical stimulation disturbances can be reduced by patient model partitioning. Two subsets resulting from partitioning are sufficient to significantly reduce the effects of the inter-patient variability in sensitivity of the anesthetic drug. Simulations have been done to show the improvement using two subsets compared to the nominal controller (used in previous research and clinical trials) and to a controller that were optimized over the model set.

Partitioning based on the $v$-gap metric resulted in satisfactory attenuation of surgical disturbances for both children and adults. As long as a full credible dynamic model is not possible to identify during clinical practice, this method is not possible to implement, even though the resulting attenuation was the largest of all evaluated methods.

For clinical practice, partitioning based on demographics was proposed. This thesis has come to the conclusion that age is a relevant parameter to describe difference in drug sensitivity for children. A controller should be chosen depending on "low" and "high" age. For adults, weight is a suitable parameter when choosing controller. These parameters have the advantage of being easy to measure and therefore this proposed method using demographics is easy to implement in clinical practice. The resulting controllers from partitioning for children and adults have been synthesized in this thesis with acceptable performance and preserved robustness.

The proposed method in this thesis of choosing a controller based on age for children and weight for adults reduces the effects of the inter-patient variability. This results in safer closed-loop controlled anesthesia with less risk of under- and overdosage. 


\section{Bibliography}

Åström, K. J. and T. Hägglund (2006). Advanced PID Control. ISA - The Instrumentation, Systems and Automation Society, North Carolina, USA. ISBN: 9781-55617-942-6.

Åström, K. and R. Murray (2008). Feedback Systems : An Introduction for Scientists and Engineers. Princeton University Press, Princeton and Oxford. ISBN: 9781400828739.

Bibian, S., G. A. Dumont, M. Huzmezan, and C. R. Ries (2006). "Patient variability and uncertainty quantification in clinical anesthesia. Part I PKPD modeling and identification". In: pp. 549-554. DOI: 10 . 3182 / 20060920 - 3 - FR - 2912 . 00097.

Bibian, S., C. R. Ries, X. Huzmeman, and G. Dumont (2005). "Introduction to automated drug delivery in clinical anesthesia". European Journal of Control 11, pp. 535-557. DOI: $10.3166 /$ j j. 11.535-557.

Bryan, J. (2004). "Problems in gene clustering based on gene expression data". Journal of Multivariate Analysis 90:1. Special Issue on Multivariate Methods in Genomic Data Analysis, pp. 44-66. DOI: 10.1016/j . jmva. 2004.02.011.

Dumont, G., L. Ngai, C. Petersen, T. Chazot, and M. Fischler (2011). "Closedloop administration of propofol guided by the neurosense: clinical evaluation using robust proportional- integral-derivative design". In: American Society of Anesthesiologists (ASA) Annual Meeting 2011. (Oct. 18, 2011). Chicago, IL, USA.

Hume, R. (1966). "Prediction of lean body mass from height and weight". Journal of Clinical Pathology 19:4, pp. 389-391. DOI: 10.1136/jcp.19.4.389.

Kandasamy, W. and F. Smarandache (2012). Dual Numbers. ZIP Publisher, Columbus, OH, USA. ISBN: 978-1599731841.

Kirkpatrick, S., C. D. Gelatt, and M. P. Vecchi (1983). "Optimization by simulated annealing”. Science 220:4598, pp. 671-680. DOI: 10 . 1126/science . 220 . 4598.671 . 
Kraft, D. (1988). A Software Package for Sequential Quadratic Programming. Technical Report DFVLR-FB 88-28. Wiss. Berichtswesen d. DFVLR, Germany.

Liu, N., T. Chazot, S. Hamada, A. Landais, N. Boichut, C. Dussaussoy, B. Trillat, L. Beydon, E. Samain, D. Sessler, and M. Fischler (2011). "Closed-loop coadministration of propofol and remifentanil guided by bispectral index: a randomized multicenter study". Anesthesia and analgesia 112, pp. 546-57. DOI: 10.1213/ANE.0b013e318205680b.

Manning, C. D., P. Raghavan, and H. Schütze (2008). Introduction to Information Retrieval. Cambridge University Press, New York, NY, USA. ISBN: 978-0-52186571-5.

Mayo, C. W., R. G. Bickford, and J. Faulconer Albert (1950). "Electroencephalographically controlled anesthesia in abdominal surgery". Journal of the American Medical Association 144:13, pp. 1081-1083. DOI: 10.1001/jama. 1950. 02920130033008.

Ng, A. Y., M. I. Jordan, and Y. Weiss (2001). "On spectral clustering: analysis and an algorithm". In: Proceedings of the 14th International Conference on Neural Information Processing Systems: Natural and Synthetic. (Dec. 3-8, 2001). NIPS'01. MIT Press, Vancouver, BC, Canada, pp. 849-856. DOI: 10.1.1.19. 8100.

Ranta, S., R. Laurila, J. Saario, T. Ali-Melkkila, and M. Hynynen (1998). "Awareness with recall during general anesthesia: incidence and risk factors". Anesthesia \& Analgesia 86, pp. 1084-1089. DOI: 10 . 1213/00000539-19980500000035.

Sadati, N., M. Hosseinzadeh, and G. Dumont (2018). "Multi-model robust control of depth of hypnosis". Biomedical Signal Processing and Control 40, pp. 443453. DOI: $10.1016 / \mathrm{j}$. bspc. 2017.10 .003 .

Shi, J. and J. Malik (2000). "Normalized cuts and image segmentation". IEEE Trans. Pattern Anal. Mach. Intell. 22:8, pp. 888-905. DOI: 10 . 1109/34 . 868688.

Sigl, J. C. and N. Chamoun (1994). "An introduction to bispectral analysis for electroencephalogram". Journal of Clinical Monitoring 10, pp. 392-404. DOI: 10.1007/BF01618421.

Soltesz, K., K. Heusden, G. Dumont, T. Hägglund, C. Petersen, N. West, and M. Ansermino (2012). "Closed-loop anesthesia in children using a PID controller: a pilot study". In: IFAC Proceedings Volumes. (Mar. 28, 2012). Vol. 45:3. Brescia, Italy, pp. 317-322. DOI: 10.3182/20120328-3-IT-3014.00054.

Soltesz, K., K. van Heusden, M. Hast, J. Ansermino, and G. Dumont (2016). “A synthesis method for automatic handling of inter-patient variability in closedloop anesthesia". In: Proceedings of the 2016 American Control Conference. (July 6-8, 2016). Institute of Electrical and Electronics Engineers Inc., Boston, USA, pp. 4877-4882. DOI: 10.1109/ACC. 2016.7526125. 
Struys, M. M. R. F., T. De Smet, L. F. M. Versichelen, S. Van de Velde, R. Van den Broecke, and E. P. Mortier (2001). "Comparison of Closed-loop Controlled Administration of Propofol Using Bispectral Index as the Controlled Variable versus "Standard Practice" Controlled Administration". Anesthesiology: The Journal of the American Society of Anesthesiologists 95:1, pp. 6-17.

Struys, M. M. R. F., T. D. Smet, S. Greenwald, A. R. Absalom, S. Bingé, and E. P. Mortier (2004). "Performance evaluation of two published closed-loop control systems using bispectral index monitoring: a simulation study." Anesthesiology 100 3, pp. 640-7. DOI: 10 . 1097/00000542-200403000-00026.

van Heusden, K., J. Ansermino, K. Soltesz, S. Khosravi, N. West, and G. Dumont (2013). "Quantification of the variability in response to propofol administration in children". IEEE Transactions on Bio-medical Electronics 60:9, pp. 25212529. DOI: 10.1109/TBME. 2013.2259592.

van Heusden, K., M. Ansermino, and G. Dumont (2017). "Robust MISO Control of Propofol-Remifentanil Anesthesia Guided by the NeuroSENSE Monitor". IEEE Transactions on Control Systems Technology PP, pp. 1-13. DOI: 10.1109 / TCST . 2017.2735359.

van Heusden, K., G. A. Dumont, K. Soltesz, C. L. Petersen, A. Umedaly, N. West, and J. M. Ansermino (2014). "Design and clinical evaluation of robust PID control of propofol anesthesia in children". IEEE Transactions on Control Systems Technology 22:2, pp. 491-501. DOI: 10.1109/tcst. 2013. 2260543.

Vinnicombe, G. (2001). Uncertainty and feedback. Ho loop-shaping and the v-gap metric. Imperial College Press, London, UK, pp. 104-123. ISBN: 1-86094-163$\mathrm{X}$.

von Luxburg, U. (2007). "A tutorial on spectral clustering". Statistics and Computing 17:4, pp. 395-416. DOI: 10.1007/s11222-007-9033-z.

Ward, J. H. (1963). "Hierarchical grouping to optimize an objective function". Journal of the American Statistical Association 58:301, pp. 236-244. DOI: 10 . 1080/01621459.1963.10500845.

Zikov, T., S. Bibian, G. Dumont, M. Huzmezan, and C. R Ries (2006). "Quantifying cortical activity during general anesthesia using wavelet analysis". IEEE transactions on bio-medical engineering 53, pp. 617-32. DOI: 10.1109/TBME . 2006.870255 . 


\section{Nomenclature}

\section{Symbols}

$\delta \quad$ Parameter in estimation of delay from induction data

$\delta_{v} \quad$ The $v$-gap

$\eta \quad$ Number of open right half plane poles

$\gamma \quad$ Nonlinearity parameter in Hill function

$\kappa \quad$ Point-wise gap between two transfer functions

$\mathscr{H}_{\infty} \quad$ Vector norm

$\mathscr{L}_{2} \quad$ Euclidean norm

$v \quad$ Greek letter $\mathrm{Nu}$

A Affinity/similarity matrix

C Dissimilarity matrix

$C_{e} \quad$ Concentration at effect site $\quad \mathrm{kg} / \mathrm{m}^{3}$

$\begin{array}{lll}C_{p} & \text { Blood plasma concentration } \quad \mathrm{kg} / \mathrm{m}^{3}\end{array}$

$D \quad$ Degree matrix

d Surgical stimulation disturbance

$d_{c} \quad$ Chordal distance

E Clinical effect

$e \quad$ Control error, $e=r-y$

$E_{0} \quad$ Clinical effect in absence of drug $\quad \approx 100$ 
$E C_{50} \quad 50 \%$ clinical effect

I Identity matrix

$k \quad$ Parameter in PD model

$k_{d} \quad$ Derivative gain

$k_{i} \quad$ Integral gain

$k_{p} \quad$ Proportional gain $\mathrm{mg} / \mathrm{kg} / \mathrm{min} / \mathrm{WAV}_{\mathrm{CNS}}$

$K S \quad$ Transfer function from $n$ to $u$

L Unnormalized graph Laplacian matrix

$l \quad$ Linkage, distance between two linked clusters

$L_{\text {sym }} \quad$ Normalized graph Laplacian matrix

$M_{S} \quad$ Maximum sensitivity

$M_{T} \quad$ Maximum complementary sensitivity

$M_{y} \quad$ Maximum undershoot

$M_{K S} \quad$ Maximum value of $K S$

$n \quad$ Measurement noise

$r \quad$ Setpoint for $y$

$S \quad$ Sensitivity

$s \quad$ Laplace transform variable

$T \quad$ Complementary sensitivity

$t \quad$ Parameter in estimation of delay from induction data

$T_{d} \quad$ Parameter in PD model, time delay

$T_{f} \quad$ Filter parameter

$u \quad$ Propofol infusion rate

$\mathrm{mg} / \mathrm{kg} / \mathrm{min}$

$y \quad$ Measured clinical effect in $\mathrm{WAV}_{\mathrm{CNS}}$ units

$z \quad$ Complex number

$z^{*} \quad$ Complex conjugate of $z$ 
Bibliography

\section{Acronyms}

BIS Bispectral index

DOH Depth of hypnosis

EEG Electroencephalogram

LBM Lean body mass

LTI Linear time invariant system

PD Pharmacodynamic (model)

PID Controller with proportional, integral and derivative part

PK Pharmacokinetic (model)

PKPD Combined PK and PD (model)

SLSQP Sequential Least Squares Programming. Local optimization method $\mathrm{WAV}_{\mathrm{CNS}}$ Wavelet-based index for measure of consciousness in anesthesia wno Winding number 


\begin{tabular}{|c|c|c|c|}
\hline \multirow{3}{*}{\multicolumn{2}{|c|}{$\begin{array}{l}\text { Lund University } \\
\text { Department of Automatic Control } \\
\text { Box } 118 \\
\text { SE-221 } 00 \text { Lund Sweden }\end{array}$}} & \multicolumn{2}{|c|}{$\begin{array}{l}\text { Document name } \\
\text { MASTER'S THESIS }\end{array}$} \\
\hline & & \multicolumn{2}{|l|}{$\begin{array}{l}\text { Date of issue } \\
\text { May } 2019\end{array}$} \\
\hline & & \multicolumn{2}{|l|}{$\begin{array}{l}\text { Document Number } \\
\text { TFRT-6080 }\end{array}$} \\
\hline \multicolumn{2}{|c|}{$\begin{array}{l}\text { Author(s) } \\
\text { Ylva Wahlquist }\end{array}$} & \multicolumn{2}{|c|}{$\begin{array}{l}\text { Supervisor } \\
\text { Kristian Soltesz, Dept. of Automatic Control, Lund } \\
\text { University, Sweden } \\
\text { Rolf Johansson, Dept. of Automatic Control, Lund } \\
\text { University, Sweden (examiner) }\end{array}$} \\
\hline \multicolumn{4}{|c|}{$\begin{array}{l}\text { Title and subtitle } \\
\text { Individualized closed-loop anesthesia through patient model partitioning }\end{array}$} \\
\hline \multicolumn{4}{|c|}{$\begin{array}{l}\text { Abstract } \\
\text { This master thesis project proposes methods for individualizing closed-loop controlled anesthesia. } \\
\text { One of the largest challenges with closed-loop anesthesia is the variation between patients in the } \\
\text { sensitivity to the anesthetic drug, here propofol. Due to limited excitation in the process dynamics } \\
\text { together with a high measurement noise level is it not possible to determine a full reliable model } \\
\text { describing a patient's dynamics online. The method used here for minimizing the effects of inter- } \\
\text { patient variability was through patient model partitioning of children and adult models. Partitioning } \\
\text { was based on similarity measures between patients, for example age, weight and applied to a dynamic } \\
\text { model describing each patient. For each subset resulting from partitioning, an optimal PID controller } \\
\text { has been synthesized. This thesis has shown that the effects of inter-patient variability can be reduced } \\
\text { using partitioning into two subsets. More subsets did not result in a significant reduction. Partitioning } \\
\text { based on n-gap between patient models resulted in the best attenuation of surgical stimulation } \\
\text { disturbances. Partitioning based on age for children and weight for adults reduces the impact from } \\
\text { surgical stimulation were proposed for clinical practices. These methods are easy to implement } \\
\text { because the demographics are known beforehand and does not depend on actual measurements during } \\
\text { the anesthesia. The results are substantiated by simulations and calculations of achieved attenuation } \\
\text { with acceptable performance and preserved robustness. }\end{array}$} \\
\hline \multicolumn{4}{|l|}{ Keywords } \\
\hline \multicolumn{4}{|c|}{ Classification system and/or index terms (if any) } \\
\hline \multicolumn{4}{|c|}{ Supplementary bibliographical information } \\
\hline \multicolumn{3}{|l|}{$\begin{array}{l}\text { ISSN and key title } \\
0280-5316\end{array}$} & $I S B N$ \\
\hline $\begin{array}{l}\text { Language } \\
\text { English }\end{array}$ & $\begin{array}{l}\text { Number of pages } \\
1-58\end{array}$ & \multirow{2}{*}{\multicolumn{2}{|c|}{ Recipient's notes }} \\
\hline Security classificat & & & \\
\hline
\end{tabular}

http://www.control.lth.se/publications/ 